$281.11146 \cdot 5$

\title{
Verhandlungen der Deutschen Gesellschaft für Neurologie
}

58. Tagung

Jahrestagung vom 19.-22. September 1984 in Heidelberg 


\section{Kardiovaskuläre}

Erkrankungen und

Nervensystem

Neurotoxikologie

Probleme

des Hirntodes

Herausgegeben von

H. Gänshirt, P. Berlit und G. Haack

Mit 216 Abbildungen und 133 Tabellen

Springer-Verlag

Berlin Heidelberg New York Tokyo 
Prof. Dr. Heinz GänSHIRT

Neurologische Klinik der Universität

Voss-Straße 2, 6900 Heidelberg

Privatdozent Dr. Peter Berlit

Neurologische Klinik Mannheim

der Universität Heidelberg

Theodor-Kutzer-Ufer, 6800 Mannheim

Dr. GABRIELE HAACK

Neurologische Klinik der Universität

Voss-Straße 2, 6900 Heidelberg

ISBN 3-540-13949-4 Springer-Verlag Berlin Heidelberg New York Tokyo ISBN 0-387-13949-4 Springer-Verlag New York Heidelberg Berlin Tokyo

CIP-Kurztitelaufnahme der Deutschen Bibliothek

Kardiovaskuläre Erkrankungen und Nervensystem, Neurotoxikologie, Probleme des Hirntodes: [Jahrestagung vom 19.-22. September 1984 in Heidelberg] / hrsg. von H. Gänshirt ...Berlin ; Heidelberg ; New York ; Tokyo : Springer, 1985.

(Verhandlungen der Deutschen Gesellschaft für Neurologie ; 3) (... Tagung / Deutsche

Gesellschaft für Neurologie ; 58)

ISBN 3-540-13949-4 (Berlin ...)

ISBN 0-387-13949-4 (New York ...)

NE: Gänshirt, Heinz [Hrsg.] ; Deutsche Gesellschaft für Neurologie: Verhandlungen der Deutschen ...; Deutsche Gesellschaft für Neurologie: ... Tagung

Das Werk ist urheberrechtlich geschützt. Die dadurch begründeten Rechte, insbesondere die der Übersetzung, des Nachdrucks, der Entnahme von Abbildungen, der Funksendung, der Wiedergabe auf photomechanischem oder ähnlichem Wege und der Speicherung in Datenverarbeitungsanlagen bleiben, auch bei nur auszugsweiser Verwertung, vorbehalten.

Die Vergütungsansprüche des $\S 54$, Abs. 2 UrhG werden durch die „Verwertungsgesellschaft Wort". München, wahrgenommen.

(c) Springer-Verlag Berlin Heidelberg 1985

Printed in Germany

Die Wiedergabe von Gebrauchsnamen, Handelsnamen, Warenbezeichnungen usw. in diesem Werk berechtigt auch ohne besondere Kennzeichnung nicht zu der Annahme, daß solche Namen im Sinne der Warenzeichen- und Markenschutz-Gesetzgebung als frei zu betrachten wären und daher von jedermann benutzt werden dürften.

Produkthaftung: Für Angaben über Dosierungsanweisungen und Applikationsformen kann vom Verlag keine Gewähr übernommen werden. Derartige Angaben müssen vom jeweiligen Anwender im Einzelfall anhand anderer Literaturstellen auf ihre Richtigkeit überprüft werden.

Offsetdruck und Bindearbeiten: Beltz Offsetdruck, Hemsbach/Bergstr.

Verantwortlich für den Anzeigenteil: M. Olle, Kurfürstendamm 237, D-1000 Berlin 15

$2125-3130 / 543210$ 


\section{Inhaltsverzeichnis}

Verzeichnis der Autoren und Vortragenden $\ldots \ldots \ldots \ldots \ldots \ldots \ldots \ldots$ XXI

Vorwort

XLIII

Verleihung der Wilhelm-Erb-Denkmünze.

Laudatio auf Professor Dr.med. Stefan Környey

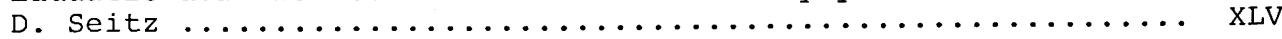

Verleihung des Hugo-Spatz-Preises.

Laudatio auf Dr. Terry Jones

W. - D. Heiss ...........................................

Verleihung des Parkinson-Preises Frosst-Pharma.

Laudatio auf Professor Dr.med. P. Riederer

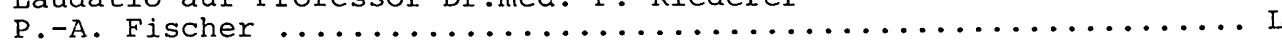

I. Kardiovaskuläre Erkrankungen und Nervensystem

Valvular Diseases in Relation to Cerebral Ischemia

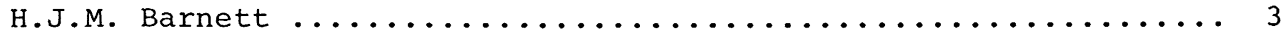

Herzrhythmusstörungen und neurologische Komplikationen

$\mathrm{J}$. Senges und $\mathrm{w}$. kübler $\ldots \ldots \ldots \ldots \ldots \ldots \ldots \ldots \ldots \ldots \ldots \ldots \ldots \ldots$

Endokarditis und ihre Auswirkung auf das Zentralnervensystern

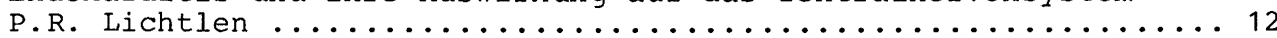

Kardiomyopathien

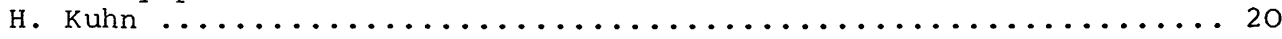

Herz, Hypertonie und hypertensive Krisen

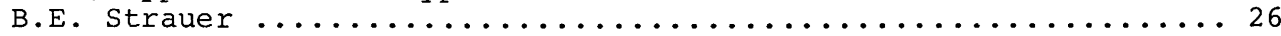

Angeborene Angiokardiopathien

D. Wolf .................................... 44

Zur Aussagekraft der eindimensionalen Echokardiographie bei

Schlaganfallpatienten

H. Henningsen, C. Kessler, R. Reuther, G. Opitz und M. Hüfner ... 49

Nicht-invasive Diagnostik nicht-stenosierender extrakranieller

Karotisläsionen mit der Ultraschall-Dopplersonographie

M. Hennerici, M. Daffertshofer, G. Esser und A. Aulich ........ 54 
Transkranielle Dopplersonographie: Ein neues Ultraschallverfahren zur Initial- und Verlaufdiagnostik intrakranieller Stenosen der A. carotis interna, A. cerebri media und der Vertebrobasilararterien

E.B. Ringelstein, G. Korbmacher, F. Wulfinghoff und H. Zeumer ... 60

Zum Aussagewert von Duplex-Scan-Untersuchungen der Karotiden bei Patienten mit zerebro-vaskulären Erkrankungen

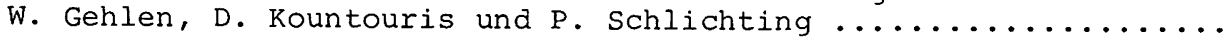

Ergebnisse von Dopplersonographie-Untersuchungen der hirnversorgenden Arterien an einer Klinik für Rehabilitation und Diagnostik kardiologischer, neurologischer und angiologischer Erkrankungen

im Zeitraum von neun Monaten (1.4.-31.12.1983)

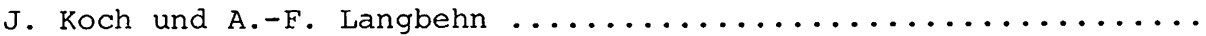

Spontanverlauf extrakranieller Stenosen und Verschlüsse der hirnversorgenden Arterien: Zwischenergebnisse einer prospektiven

Studie

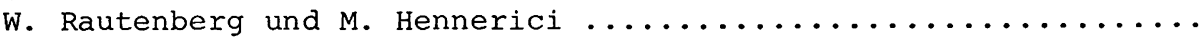

Spontanverlauf arteriosklerotischer Plaques der A. carotis: Progredienz und Regredienz

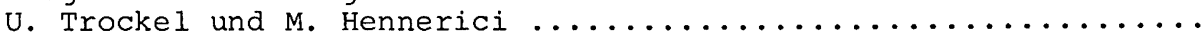

Bilaterale traumatische Karotisverschlüsse - Diagnostik,

Verlauf und Prognose

U. Schultz, M. Kütemeyer und H.W. Kölmel $\ldots \ldots \ldots \ldots \ldots \ldots \ldots$

Frühe akustisch evozierte Potentiale (FAEP) bei Basilaristhrombose

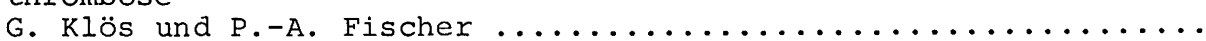

Späte kognitionsabhängige Komponenten evozierter Potentiale

(P 300) bei asymptomatischen extrakraniellen Gefäßprozessen

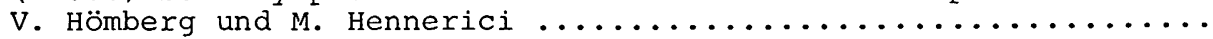

Intraoperatives Monitoring in der Karotis-Chirurgie mit somatosensorisch evozierten Potentialen (SEP)

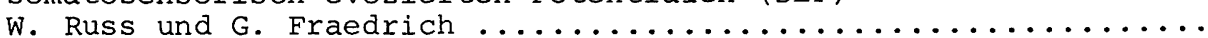

Der Nachweis intrakardialer Emboliequellen bei Schlaganfallpatienten mit Hilfe der 111 In-Plättchenszintigraphie

C. Kessler, R. Reuther, H. Henningsen, B. Kimmig und M. Rösch .. 102

Mitralklappenprolaps und Thrombozytenfunktionsstörungen bei

jüngeren Patienten mit zerebralen Ischämien

G. Krämer, M. Tophof, B. Henkel und R. Erbel

Migräne, Mitralklappenprolaps und Thrombozytenaggregation V. Pfaffenrath, G. Autenrieth, U. Kellhammer, W. Pöllmann und

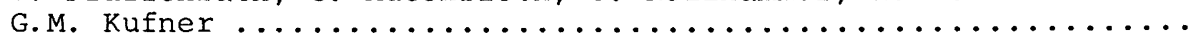

Zur Messung zirkulierender Plättchenaggregate bei Patienten mit zerebro-vaskulären Erkrankungen

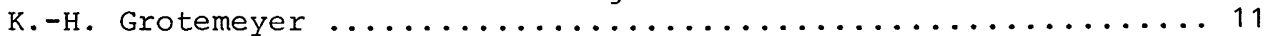

Die regionale Gehirndurchblutung beim Hirninfarkt infolge kardiologischer Erkrankungen

A. Hartmann 
Neue Möglichkeiten der quantitativen Hirndurchblutungsmessung mit dem kurzlebigen Isotop $195 \mathrm{~m}_{\mathrm{Gold}}$

P. Lindner, O. Nickel, D. Eissner, P. Ullrich, K. Hahn,

B. Pfeiffer, S. Wende und H.C. Hopf $\ldots \ldots \ldots \ldots \ldots \ldots \ldots \ldots \ldots$

Positronen-Emissions-Tomographie des Gehirns: Neue Ergebnisse bei Hirngefäßerkrankungen

S. Herold, J.M. Gibbs, R.J.S. Wise, R.S.J. Frackowiak und

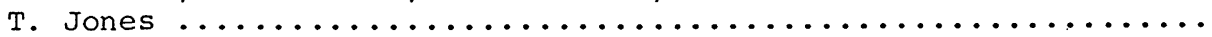

Gehirndurchblutung und zerebrales Blutvolumen bei Karotisverschlüssen und -stenosen

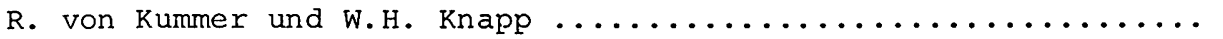

Die analoge intravenöse Subtraktionsangiographie - Eine

Alternative zur DSA?

W.-D. Möller, H.-L. Poser und K. Wolschendorf ............ 133

Computergestützte epidurale Hirndruckmessung beim

raumfordernden Insult

A. Haaß, T. Madler und $\mathrm{K}$. Schimrigk ....................

Zentrale und oberflächliche Hemisphären-Infarkte. (E.ine

Gegenüberstellung klinischer, computertomographischer und

angiographischer Befunde)

O. Busse, M. Kaps, D. Schwirz und o. Hoffmann ................142

Liquorenzyme als Gewebszerfallmarker in der Frühiiagnostik

ausgeprägter Hirninfarkte

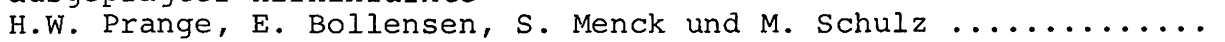

Diagnose der kardialen Hirnembolie aus neurologischer Sicht

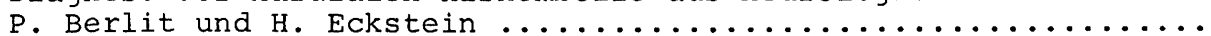

Herzrhythmusstörungen bei transitorisch-ischämischen Attacken

G. Freier, T. Stober, S. Sen, T. Anstätt und K. Schimrigk ..... 158

Juvenile Insulte: Pathogenese und Verlaufsbeobachtungen

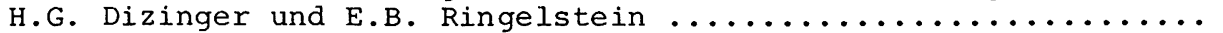

Die isolierte supraklinoidale Einengung der A. carotis interna

H.L. Lagrèze, A. Hartmann, J. Wappenschmidt, R. Ries und

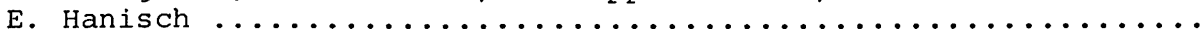

Prognostische Beurteilung venöser und arterieller zerebraler Durchblutungsstörungen (Eine vergleichende Untersuchung)

K.M. Einhäupl, J. Rieder, C.H. Garner, W. Meister und W. Keeser

Vertebro-basiläre Verschlüsse: Klinische Syndrome und Verläufe unter nicht invasiver Therapie

L. Lachenmayer, R.W.C. Janzen, D. Kühne, K. Kunze, C. Meier

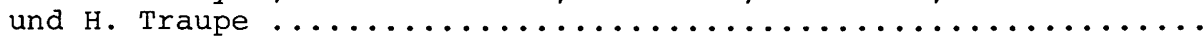

Diagnostik, Therapie und Prognose postischämischer neuronaler

Dysfunktion

H. Schoeppner, L. Rolf und M. Hoke ................ 180

Vertikale Blickparese und Thalamische Demenz

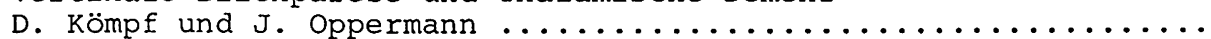


Progrediente Myoklonien als Symptom eines Hirnstamm- und Kleinhirninfarktes

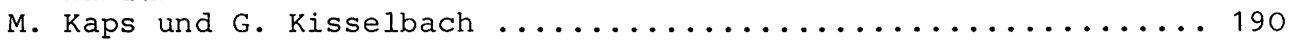

Transiente Bewußtseinsstörungen bei ischämischen Läsionen im Thalamus

E. Schneider, E. Schönbrunn, D. Vonofakos und M. Ruß ........ 192

Kasuistischer Beitrag zur ätiologischen Klärung einer schwer zu diagnostizierenden "Ohnmacht"

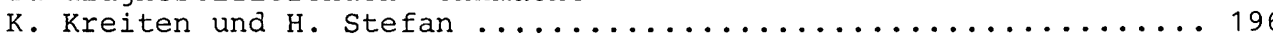

Myokardläsionen bei Patienten mit neurogenen EKG-Veränderungen

T. Anstätt, K.H. Merkel, T. Stober, S. Sen, G. Freier und

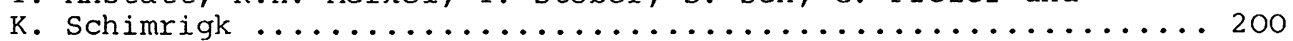

Lungenfunktion und kardiologische Untersuchungen bei

Friedreich-Ataxie

L. Burger, H. Walle, M. Müller, G. Rettig und T. Stober ...... 204

Untersuchungen zur autonomen Neuropathie bei Patienten mit

diabetischer Polyneuropathie und Patienten mit chronischem

Alkoholabusus mittels automatischer Analyse der Herzfrequenzvariabilität

B. Buchinger, G. Herrmann und M. Kaps ................ 208

Kardiovaskuläre Erkrankungen und Parkinsonismus

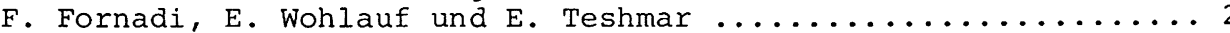

Uhthoff-Phänomen bei nicht demyelinisierenden ErkrankungenVaskulär bedingte passagere Leitungsverzögerung im N. opticus

M. Pause, V. Hömberg und M. Hennerici ................ 215

Zum Verlaufstyp spinovaskulärer syndrome

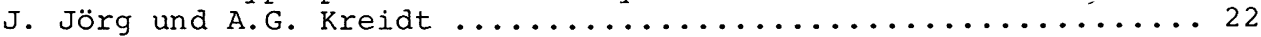

Korrelationsuntersuchungen zwischen Kreislaufregulations-

störungen vor und nach Lumbalpunktionen und postpunktionellen Beschwerden

W. Greulich, N. Ikonomou, D. Kountouris und W. Gehlen

Alkohol als eine der Ursachen von Übergewicht, "essentiellem"

Bluthochdruck, Hyperlipidämie und Schlaganfall

H.H. Kornhuber, G. Lisson und L. Suschka-Sauermann ..........

Störung der Blut-Liquor-Schranke für Proteine und Enhancement

nach Kontrastmittelgabe im CT bei ischämischen zerebralen

Infarkten

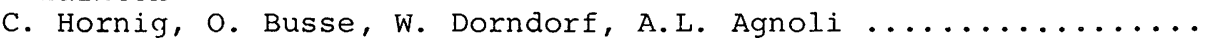

Arteriitis temporalis als Notfallsituation

K.-F. Druschky, A. Barner, W.P. Kaschka, F.-H. Meythaler und

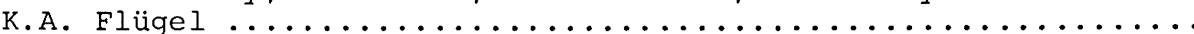

Klinische Aspekte der luetischen Angiopathie

T. Alexopoulos, K. Kunze und A. Müller-Jensen ........... 239

Zur Indikation, Methodik und Wertigkeit der immunhistologischen

Diagnostik beim Hirninfarkt

P. Berlit, C. Kessler, C. Carls und $\mathrm{K} .-\mathrm{H}$. Krause ...........243

Granulomatöse Angiitis des ZNS und ihre klinische Relevanz

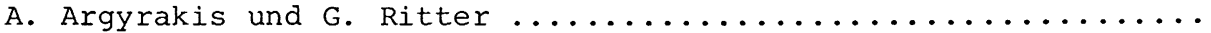


Multiinfarktsyndrom bei jüngeren Patienten mit Livedo racemosa: Vaskulitis oder Endokarditis?

G. Reifschneider, F. v. Baumgarten, H.G. Mertens, B. Maisch und

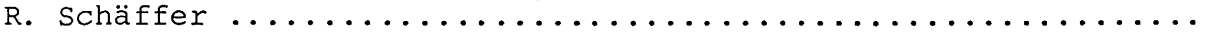

Livedo racemosa mit zerebraler Beteiligung (Ehrmann-SneddonSyndrom)

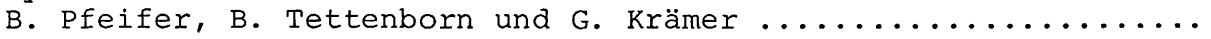

Hypertonie und Nachblutungsrisiko bei Subarachnoidalblutungen

T. Stober, T. Anstätt, S. Sen, H. Emde, U. Metzger und

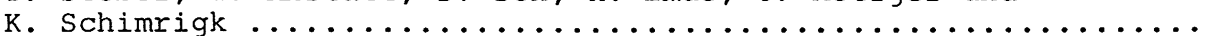

Bedeutung von Thromboxan $\mathrm{A}_{2}$ für die Pathogenese des zerebralen Vasospasmus nach Subarachnoidalblutung

R.L. Haberl, E. Hiller, H. Riess, J. Rieder, M. Prosiegel und

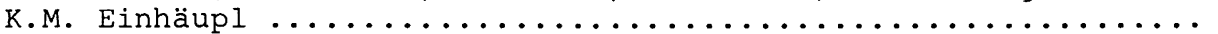

Einfluß kardiovaskulärer Risikofaktoren auf den Verlauf spontaner intrazerebraler Hämatome

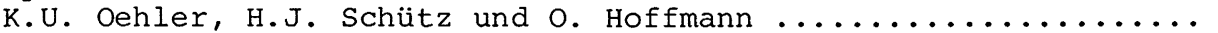

Verlauf und Prognose von 47 Patienten mit spontanen Thalamushämatomen

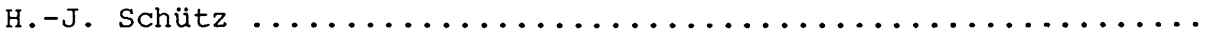

Klinisch-neurologische Aspekte zerebellärer Hämangioblastome

(Lindau-Tumor)

A. Müller-Jensen und $\mathrm{W} . \mathrm{H}$. Zangemeister $\ldots \ldots \ldots \ldots \ldots \ldots \ldots$

Schrittmacherbehandlung bradykarder Herzrhythmusstörungen bei akuter zerebraler Ischämie

H. Bewermeyer, A. Wandel, V. Hossmann und W.-D. Heiss .........

Zur Herzschrittmacherindikation bei Polyradikulitis: Ein

standardisiertes Verfahren für den Bulbusdruckversuch

D. Englert, F.J. von Baumgarten, G. Gunreben, G. Reifschneider,

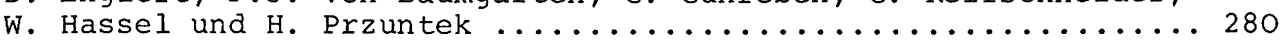

Hypersensitiver Karotissinus-Reflex aus neurologischer Sicht

K. F. Druschky, J. Kotzian, K. - D. Preiss und R. Leutschaft .....

Zerebrale Leitsymptome beim Karotis-Sinussyndrom und ihre

Beeinflussung durch Schrittmacherimplantation

J.R. Bayerl, W. Busch, W. Kollmeier und H. Schworm ...........

Klinische, hirnelektrische und psychopathologische Befunde

mit Längsschnittuntersuchungen bei Herzschrittmacherträgern

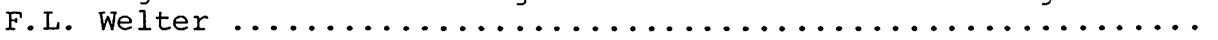

Neurologische Komplikationen nach aortokoronaren Bypassoperationen

C.R. Hornig, V. Sprengel, w. Dorndorf und R. Hoege ......... 294

Herz-Kreislauf-Beeinflussung polytraumatisierter Patienten mit Alkoholanamnese durch Fluphenazin-Dihydrochlorid

K. - W. Fritz, A. Osterhaus und $w$. Seitz $\ldots \ldots \ldots \ldots \ldots \ldots \ldots$

Pro und Contra Dextran und HAEs aus hämorheologischer sicht

A. Haaß, I. Decker, H. Jäger, W. Thönnes, H. Kiesewetter

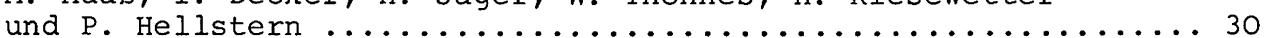


Lokale intraarterielle Fibrinolyse-Therapie der hirnversorgenden Gefäße

H. Zeumer, R. Hündgen und E.B. Ringelstein $\ldots \ldots \ldots \ldots \ldots \ldots$

Perkutane transluminale Angioplastie der supraaortalen Gefäße speziell der Vertebralisabgangsstenosen

R. Hündgen, H. Zeumer, W. Hacke und E. B. Ringelstein ........ 309

\section{Neurotoxikologie}

Organische Schäden des Nervensystems durch gewerbliche Intoxikationen und Umweltgifte

H.J. Bauer

Chemogenic Lesions, A Multifactor Concept

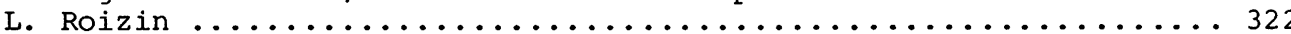

Untersuchungen zur Neurotoxizität von Arbeitsstoffen

(Ausgewählte Beispiele)

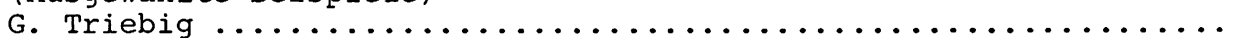

Die Hexacarbon-Neuropathien

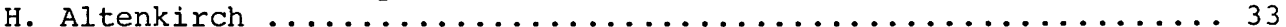

Psychorganische Residuen nach Intoxikation mit Organozinnverbindungen

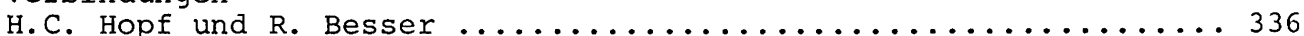

Chronische toxische Enzephalopathien nach Einwirkung organischer Lösungsmittel

P. Gregersen

Neurotoxikologische Aspekte der spanischen Massenvergiftung durch Speiseöl

H. Altenkirch und $G$. Stoltenburg-Didinger $\ldots \ldots \ldots \ldots \ldots \ldots \ldots$

Polyneuropathien durch Umwelt- und gewerbliche Gifte

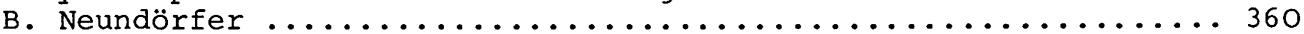

Chemische Kanzerogenese im Nervensystem

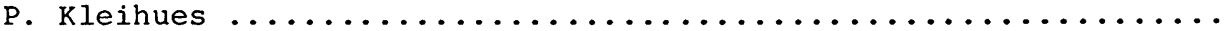

Thallium-Intoxikation - Besonderheiten in der Phänomenologie der Würzburger Vergiftungsfälle

P. Reuther, J. Epping, P. Krauseneck, H.G. Mertens und

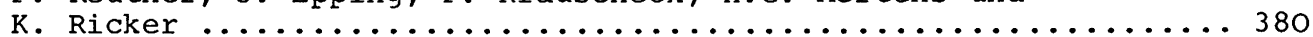

Thallium-Intoxikation - Therapie und Verlauf der würzburger Vergiftungen

J. Epping, P. Reuther, E. Heidbreder, A. Heidland, H.G. Mertens

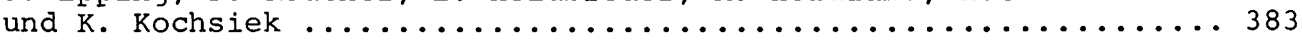

Elektrophysiologische, klinische und Serum-Spiegeluntersuchungen bei chronisch thallium-exponierten Arbeitnehmern

A. Ludolph, R. Sennhenn, C. Elger, H.P. Bertram und K. Weischer . 389

Persistierende zerebelläre Ausfälle nach Thalliumintoxikation

H. Feistner und H.J. Schütz. 
Polyneuropathien durch ungewöhnliche chronische Bleiintoxikationen

G. Reimann, R. Böhlen, P. Krauseneck, K. Ricker und R. Rohkamm . 395

Untersuchungen von VEP's, SEP's des $\mathrm{N}$. medianus und sensiblen

NLG's des $N$. medianus und $N$. radialis an bleiexponierten

Kindern

T. Ewert, U. Beginn, G. Winneke, B. Hofferberth und J. Jörg ... 399

Arsenpolyneuropathie nach Einnahme in suizidaler Absicht:

Klinik, Elektrophysiologie und Therapieversuch mit DMPS

(Dimaval)

B. Tettenborn, G. Krämer, O. Oster, H.H. Goebel und H.C. Hopf .. 403

Neurotoxizität und extrazerebrale Komplikationen unter der

Lithiumtherapie bei manisch-depressiven Erkrankungen

G. Reifschneider, D. Englert, G. Gunreben, J. Epping,

G. Fuchs und H.G. Mertens ....................... 407

Akzidentelle Manganvergiftung durch Ingestion von Kaliumpermanganat

M. Holzgraefe, W. Poser, H. Kijewski und R. Benecke ........4 412

Anästhesia dolorosa und Parkinson-Syndrom nach Trichloräthylenintoxikation - 20jährige Verlaufsbeobachtung

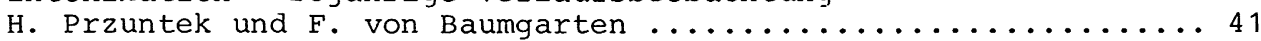

Neurotoxischer Effekt von 1-Methyl-4-phenyl-1,2,3,6-tetrahydropyridin (NMPTP) auf das nigrostriatale system und protektiver Effekt des 1-Tertiärbutyl-4,4-diphenylpiperidin (Budipin)

H. Russ, K. Henning, $H$. Eckhardt und $H$. Przuntek $\ldots \ldots \ldots \ldots \ldots$

Ösophagusfunktionsstörungen als Ursache Botulismus induzierter Dysphagie

W. A. Nix, V.F. Eckardt, G. Krämer, A. Brunier und R. Röder ....4 424

Akute porphyrische Krise mit schwerer zerebraler schädigung nach "Brigitte-Diät"

K.-F. Druschky, J. Schirmeister, P. Nerb und C. Lang

Genetisch determinierte Neurotoxizität durch Hydroxylierungsdefekt

I. Arzneimitteltoxizität durch Perhexilin: Die DA-Ratte als Tiermodell

A. Wahlländer, A. Zimmermann und $\mathrm{C}$. Meier $\ldots \ldots \ldots \ldots \ldots \ldots$

II. Morphologische Befunde am Nervensystem der Perhexilinbehandelten DA-Ratte

C. Meier und A. Wahlländer

Neurologische Folgeerkrankungen bei chronischem Alkoholismus

A. Engelhardt, B. Neundörfer, D. Claus und H. Burkowski .......4442

Blande alkohologene Hirnstammaffektionen - Untersuchungen mit akustisch evozierten Hirnstammotentialen

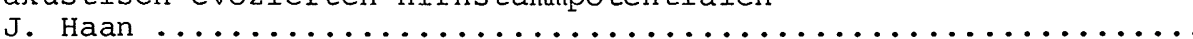

Klinischer Befund und Verlauf der sogenannten Tabak-AlkoholAmb lyopie

U. Patzhold, J. Krumsiek und $c$. Krüger $\ldots \ldots \ldots \ldots \ldots \ldots \ldots \ldots$ 
Zur Wertigkeit der konventionellen Elektromyographie im Vergleich zu Einzelfaser-EMG-Untersuchungen bei chronischem Alkoholismus

D. Kountouris, W. Greulich, S. Skondras, S. Gebes und W. Gehlen. 455

Hirnstammblutungen bei Wernicke Enzephalopathie

H.C. Braeuer, A. Müller-Jensen ...................... 458

Zentrale pontine Myelinolyse: Bericht über vier Fälle bei verschiedenen Grunderkrankungen

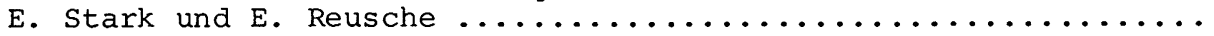

Computertomographische Untersuchungen zur Frage der Hirnatrophie durch Suchtstoffe

W. Poser, S. Poser, M. Holzgraefe, D. Roscher und A. Argyrakis.

Zur Neurotoxizität von Antiepileptika bei Langzeitbehandlung K.-H. Krause, P. Berlit und G. Kynast

Akutwirkungen von Antiepileptika auf das auditorische System des Kaninchens

T. Lenarz, $\mathrm{H}$. Henningsen und $\mathrm{C}$. Kessler $\ldots \ldots \ldots \ldots \ldots \ldots \ldots \ldots$

Die Beeinflussung der Thromboplastinzeit durch Hemmkörper unter antikonvulsiver Therapie

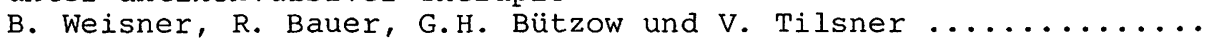

Intoxikationen mit Phenytoin während antikonvulsiver Therapie unter Berücksichtigung der pathogenetischen Bedingungen

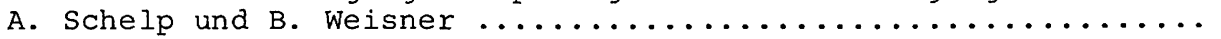

Toxizität von Zytostatika für das Zentralnervensystem

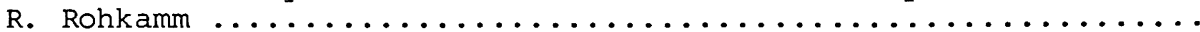

CIS-Platin: Ein onkologisches Medikament mit bemerkenswerter Neurotoxizität

C. Meier und A. Goldhirsch

Intrathekale Verträglichkeit von Cytosin-Arabinosid

P. Krauseneck, D. Dommasch, P. Dienst, U. Bogdahn, L. Kappos,

D. Seybold und H.G. Mertens ...................... 500

Verträglichkeit von Triamcinolon-Acetonid-Kristallsuspension und Antibiotika bei intrathekaler Applikation

L. Kappos, P. Krauseneck. D. Dommasch, W. Damm, U. Bogdahn und

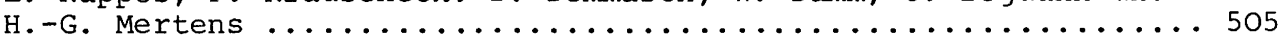

Elektrophysiologische Untersuchungen vor und nach Myelographie mit Iopamidol

U. Steller, W. -D. Möller und H. Strenge ............... 509

Einfluß verschiedenex Dialyseverfahren (Hämofiltration -Hämodialyse) auf Befindlichkeit, Konzentration, Merkfähigkeit und neurophysiologische parameter

W. $-U$. Weitbrecht, H. Schulz, F. Balck und J. Oppermann ...... 513

Toxische Myopathie durch Emetin

R. Rohkamm, G. Reimann und K. Ricker ............... 516

Beeinflussung somatosensorisch evozierter Potentiale durch

Etomidat

E. Kochs, R.D. Treede und J. Schulte am Esch ............. 520 
Neurologische Symptomatik bei Diphenhydramin- und Thymoleptikaintoxikationen

F.J. von Baumgarten, D.H. Englert, G. Reifschneider und

H. Przuntek

Malignes neuroleptisches Syndrom - Ein Fallbericht

P. Müller, $W$. Lüer, $G$. Ritter und $H$. Wismann $\ldots \ldots \ldots \ldots \ldots \ldots . \ldots 528$

III. Probleme des Hirntodes

Absence of Brain Function. - The Sign of Death

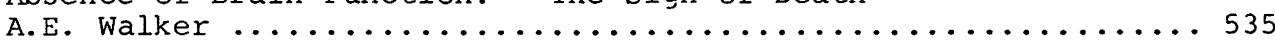

Probleme des Hirntodes

R.A. Frowein, K.-E. Richard und E. Hamel .............. 543

Pädiatrische Probleme des Hirntodes

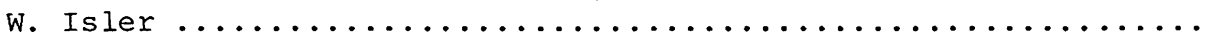

Juristische Probleme des Hirntodes

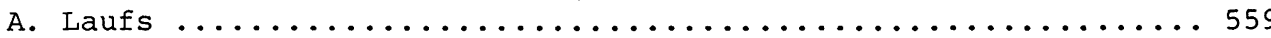

Ethische Probleme des Hirntodes

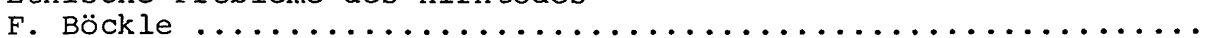

Organtransplantation

R. Pichlmayr ....................................... 570

Erfahrungen bei der Diagnose und Dokumentation des Hirntodes

H. Angstwurm, K. Einhäupl und M. Heuser ............ 577

Neurologische Symptome bei Manifestation des Hirntodes

R.W.C. Janzen, P. Hohnstädt, L. Lachenmayer, W. Rohr und

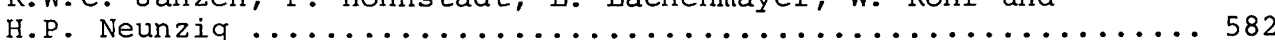

Der Hirnstammtod bei Basilaristhrombose - Eine besondere

Variante des Hirntodes?

A. Ferbert, H. Buchner, E.B. Ringelstein und w. Hacke ....... 587.

Das komplette apallische syndrom

R. Biniek, U. Schuchardt, V. Schuchardt und R. Heitmann ...... 590

Herzfrequenzvariabilität beim Hirntod

G. Schwarz, G. Pfurtscheller, V. Köpruner und w. List ....... 594

Methodische Aspekte der Ableitung evozierter Potentiale

im Koma und bei drohendem Hirntod

H. Buchner, A. Ferbert und $\mathrm{W}$. Hacke $\ldots \ldots \ldots \ldots \ldots \ldots \ldots \ldots$

Neurophysiologische und neurosonologische Verlaufsuntersuchungen beim drohenden Hirntod

W. Hacke, E.B. Ringelstein, H. Buchner, A. Ferbert und

F. Wulfinghoff

Zerebrale Fettembolie mit Langzeit-überleben. SSEP, FAEP, Blink-, Masseter-Reflex Untersuchungen. Histopathologische Befunde

E. Rumpl, R. Henn, M. Prugger, J.M. Hackl und F. Gerstenbrand .. 608 
Akustisch evozierte Hirnstamm-Potentiale in der Intensivneurologie

A. Ullrich, H. Reich, B. Riffel und M. Stöhr ............613

Wertigkeit von SEP-Untersuchungen in der Intensivneurologie

B. Riffel, A. Ullrich, M. Stöhr und w. Graser ............6 617

Perfusionssintigraphie zur Bestimmung des Hirntodes mittels eines tragbaren Strahlen-Detektor-Systems und eines Personalcomputers

K. Koppenhagen, A. Scholz, H. Schulz, J. Link, J. Mühlberg,

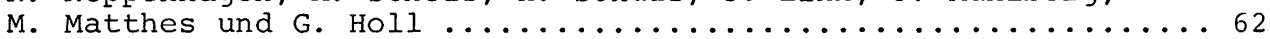

Wert der Computertomographie für die Diagnose des Hirntodes

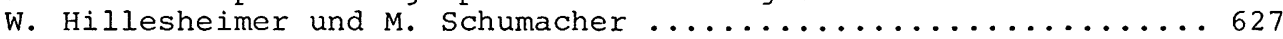

Die akute Hypodensität des Hirnstammes im CT - Zeichen des

Hirntodes?

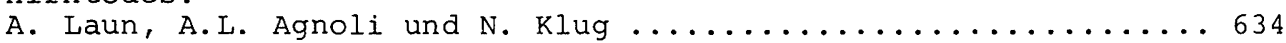

Zerebroprotektive Maßnahmen nach Herzkreislaufstillstand.

Der Einfluß hirnstoffwechselsenkender Pharmaka auf die komplette Ischämie des Gehirns

C. Krier, K.H. Jünemann, K. Wiedemann und $\mathrm{s}$. Hoyer ........6 63

Probleme bei der Vorbereitung von potentiellen Organspendern zur Mehrfachorganentnahme

J. Mühlberg, H. Schulz, R. Rohling, D.E. Rosenow, W. Wagner,

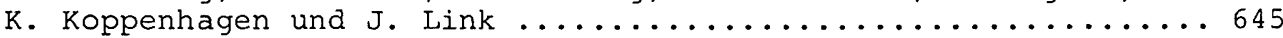

IV. Freie Themen

Somatisch evozierte Potentiale nach Stimulation des N. trigeminus bei Patienten mit Trigeminusneuralgie vor und nach operativer Dekompression

H.W. Ilsen, J. Menzel und T. Rommel $\ldots \ldots \ldots \ldots \ldots \ldots \ldots \ldots$

Das Ramsay-Hunt-Syndrom (Zoster des Ganglion geniculi)

J.-P. Malin, K. Weißenborn und H.J. Heinze .............658

Therapie des Meige-Syndroms

A. Brinkmann, F. Schumm und J. Dichgans .............. 662

Taktil ausgelöster Tinnitus

B. Conrad, R. Benecke und G. Dieckmann ..............6666

Ophthalmoplegie mit "Levator sparing" und thalamische Demenz

A. Weindl, P. Lechner, H. Gräfin von Einsiedel, G. Ochs,

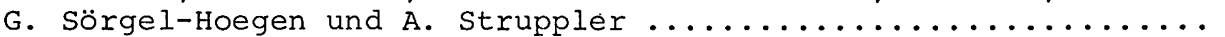

Hirnstammbeteiligung beim Fisher-Syndrom? Beobachtungen bei

fünf eigenen Fällen

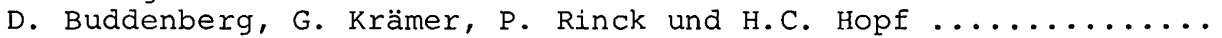

Myasthenia gravis: Klinische und immunologische Krankheitsaktivität nach Absetzen immunsuppressiver Langzeittherapie mit Azathioprin

K.V. Toyka, R. Hohlfeld, K. Heininger, B. Gerhold und

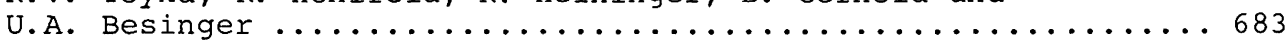


Klinische und immunologische Verlaufsuntersuchungen bei der Myasthenia gravis

W.P. Kaschka, I. Kalies, F. Skvaril, R. Hilgers, J.R. Kalden

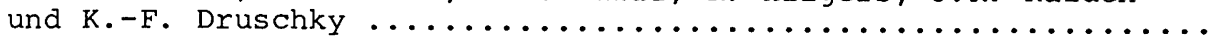

Splenektomie bei Myasthenia gravis - Ein therapeutisches Konzept?

W.E. Hofmann, P. Reuther und H.-G. Mertens ...............6 691

Klinische Pharmakokinetik von Pyridostigmin: Untersuchungen

mit einer hochdruckflüssigkeitschromatographischen Methode

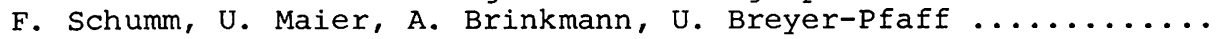

Isolierung autoimmuner T-Lymphozyten von Patienten mit

Myasthenia gravis

R. Hohlfeld, K.V. Toyka, K. Heininger und I. Kalies ..........

Nachweis von Acetylcholinrezeptor spezifischen Zellen im

Blut von Myasthenia gravis Patienten

H. Kachelries, I. Kalies, B. Koch, J.R. Kalden und K.F. Druschky 703

Monoklonale Antikörper ( $\mathrm{mAB}$ ) gegen Acetylcholine Rezeptor

(AChR) verstärken die antigenspezifische Aktivierung AChRspezifischer $T$-Zell-Linien

B.C.G. Schalke, W.E.F. Klinkert, H. Wekerle und D.S. Dwyer .... 708

HLA-Antigene bei okulärer Myasthenia gravis

A. Brinkmann, F. Schumm; C. Müller, A. Fateh-Moghadam und

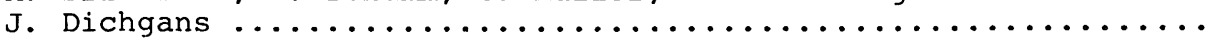

Nachuntersuchung von MS-Patienten mit über zehnjährigem

Verlauf: Vergleich von Azathioprin-behandelten und nicht immunsuppressiv-behandelten Patienten

R. Heun, L. Kappos, D. Dommasch, U. Bogdahn, P. Krauseneck

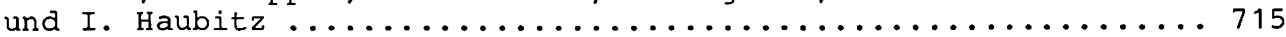

Verläufe der visuell evozierten Potentiale (VEP) bei akuter

Retrobulbärneuritis (RBN) unter Kortikoidtherapie

H. Rüttinger, W. Emser, A. Knieriem und K. Schimrigk ........719

Störungen "höherer kortikaler Funktionen" bei der multiplen

Sklerose

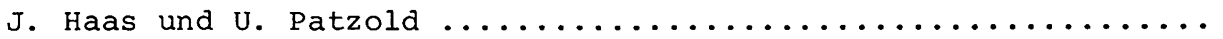

Klinische und labordiagnostische Beobachtungen bei familiärer MS

H. - P. Hartung, V. Hömberg und M. Hennerici ............ 728

Zur diagnostischen Bedeutung von long loop Reflexen bei

Multipler Sklerose

H. - H. Friedemann, J. Noth und R. Glombitza ..............733

Die spontane "Burst"-Aktivität peripherer Blutmonozyten (MO)

von MS-Patienten - Ein Parameter für die aktuelle Aktivität

des entzündlichen ZNS-Prozesses?

K. Hammann, C. Corradini, U. Dillmann, A. Fischer, A. Kleider,

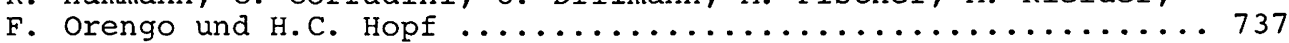

Das Komplement-Bindungsverhalten mononukleärer Zellen des peripheren Blutes von MS-Patienten mit unterschiedlichen Krankheitsphasen

K.-P. Hamann, D. von Steldern, M.P. Dierich und H.C. Hopf ..... 
Frühsommermeningoenzephalitis und Bannwarth Syndrom: Klinische und laborchemische Befunde

H.W. Pfister, K. Einhäupl, V. Preac-Mursic, B. Wilske und

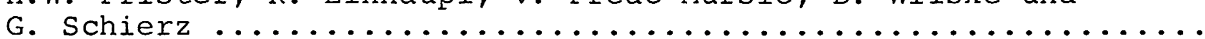

Chronische Meningomyeloradikulitis durch Ixodes-ricinus-Spirochäten - Eine der Metalues analoge Spirochätose?

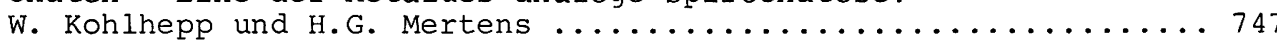

Verlauf der Herpes-simplex Enzephalitis unter AciclovirBehandlung

G. Japp, P. -A. Fischer und H. Baas ................ 750

Neurologische Komplikationen bei AIDS - 3 eigene Fälle von Toxoplasmose-Enzephalitis

W. Enzensberger, E.B. Helm, G. Japp, P.-A. Fischer und w. Stille 753

Die akute zerebelläre Enzephalitis im Erwachsenenalter.

Spezifische Augenbewegungsstörungen, Klinik, Histopathologie

D. Kömpf, H.-J. Dietrich, A. Engelhardt und B. Neundörfer .....758

Diagnostische und therapeutische Ansätze bei Tuberkulostatikaresistentem intrazerebralem Tuberkulom

U. Bogdahn, P. Reuther, B. Fleischer, L. Kappos, M. Ratzka,

F. Schick, w. Gerharts, D. Seyboldt und H.G. Mertens .........7762

Primäre meningeale Sarkomatose - Eine Kasuistik zur Differentialdiagnose des polyradikulären syndroms

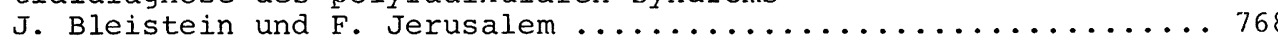

Klinische und neuropathologische Befunde bei progressiver

Sklerose (Sklerodermie)

W. Gottwald, H. Schmidt und E. Haneke ............... 770

Immunkomplex - Polyneuritis nach Kollagenase (Nucleolysin)

M. Franz, B. Pohlmann-Eden und J.-R. Bayerl ................ 775

Polyneuritis und Hepatitis

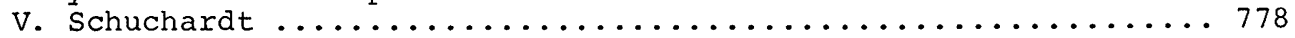

Immunologische Parameter bei 187 Patienten mit entzündlichen peripheren Neuropathien

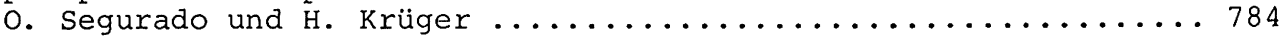

Die Bedeutung gemeinsamer Antigene im Nerven- und Immunsystem

für die Pathogenese von Immunneuropathien

G. Stoll, G. Schwendemann, K. Heininger und K.V. Toyka .......789

Ergebnisse rasterelektronenmikroskopischer Untersuchungen

an Zellen des Liquor cerebrospinalis

M. Kaps, E. Burkhardt und C. Hornig .................. 792

Isoelektrische Fokussierung (IEF) von Serum und Liquor bei

1000 neurologischen Patienten

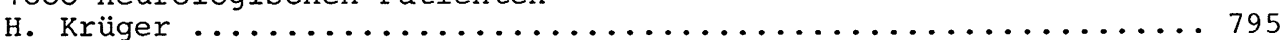

Agarose - IEF und Immunfixation von Serum und Liquor bei 50 neurologischen Patienten

U. Pickert, H. Krüger und K.W. Pflughaupt .............. 799 
Analyse von zellulären Differenzierungsantigenen auf Liquorzellen mit monoklonalen Antikörpern

R. Hohlfeld, I. Brüske-Hohlfeld, A. Schwartz und K.V. Toyka .... 803

T-Zell-Linien übertragene experimentell allergische Enzephalomyelitis ( $t-E A E)$ - vereinfachtes neuropharmakologisches Modell zur Effektivitätsmessung von Immunsuppressiva

M.E. Westarp, M. Gerlach, M.L. Vohl, H. Wekerle, P. Jutzi und

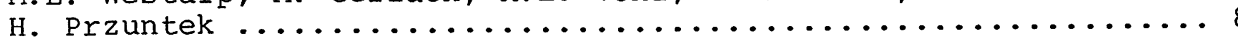

Galactocerebrosid-Experimentell-Allergische Neuritis: Tiermodell der chronischen Polyneuritis

G. Schwendemann, G. Stoll, K. Heininger, W. Köhne

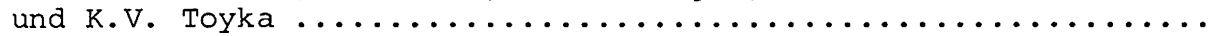

Elektrophysiologische Untersuchungen bei chronisch rezidivierender Neuritis am Kaninchen

K. Heininger, G. Stoll, G. Schwendemann, H.-P. Hartung und

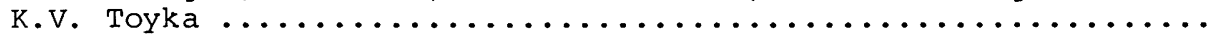

Traceruntersuchungen zur Blut-Nerv- und Blut-Hirn-Schranke

bei Mäusen mit biotinyliertem humanen und murinen IGG

R.J. Seitz, K. Heininger, G. Schwendemann, K.V. Toyka und

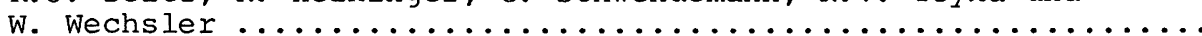

Interaktionen von Substanz $\mathrm{P}$ mit Makrophagen

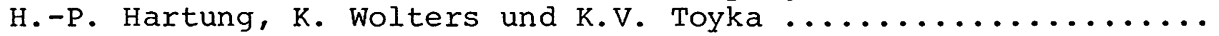

Bulbospinale hereditäre motorische Neuropathie (BSHMN).

Falldarstellung unter besonderer Berücksichtigung elektro-

physiologischer Befunde

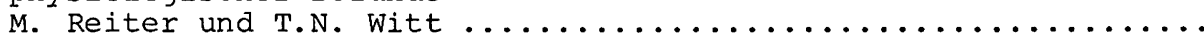

Computertomographie der Unterschenkel bei Dystrophia myotonica

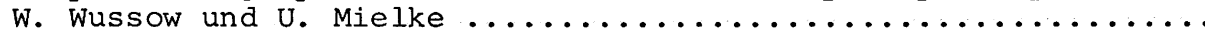

Computertomographie der Muskulatur bei neuromuskulären

Erkrankungen

G. Küther, S.o. Rodiek und A. Struppler $\ldots \ldots \ldots \ldots \ldots \ldots \ldots \ldots$

Infusionstherapie mit Thyreotropin-Releasing-Hormon bei

amyotropher Lateralsklerose

W. R. Kießling und M. Schwalbach $\ldots \ldots \ldots \ldots \ldots \ldots \ldots \ldots \ldots$

Die idiopathische lumbosakrale Plexusneuropathie. Klinische und elektrophysiologische Beschreibung eines eigenständigen Krankheitsbildes

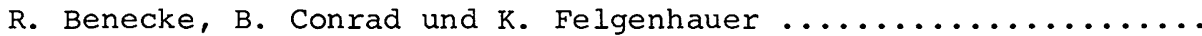

Morvan-Syndrom mit Thymom, Antikörpern gegen nikotinischen Acetylcholin-Rezeptor und elektromyographischen Zeichen einer Neuromyotonie

V. Hömberg, M. Halbach, M. Pause, R.J. Seitz, K.V. Toyka,

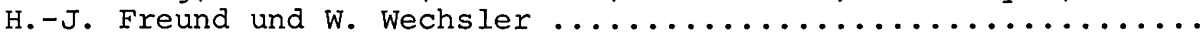

Glykogenose Typ V (Mc Ardle Syndrom) in Kombination mit anderen Störungen des Glukosestoffwechsels

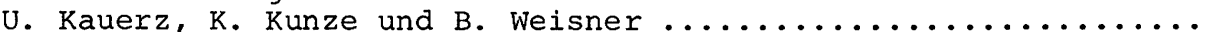

Periphere und zentralnervöse Störungen unter Folsäure- und Vitamin B6-Mangel. Eine tierexperimentelle Studie

D. Claus, R. Eggers, A. Engelhardt und B. Neundörfer .......... 
Neurogene Befunde bei adultem Saure-Maltase Mangel (SMM)

W. Schubert, P. Vogel und I. Paetzke ................ 868

Muskelrelaxation durch örtliche Iontophoreseanwendung von Suxame thoniumch lorid

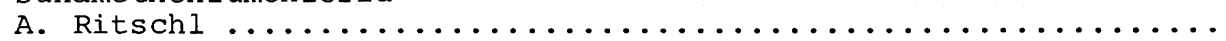

Unterschiedliche EEG-Aktivität bei Schizophrenen und Gesunden vor und während willkürlicher Fingerbewegungen

K.P. Westphal, B. Grözinger, V. Diekmann, M.M. Frech, J. Nitsch, C. Andersen, W. Scherb, K.D. Neher und H.H. Kornhuber .........

Kurzzeit-Spektral-Analyse von ereignisbezogenen EEG-Abschnitten

V. Diekmann

Charakterisierung eines Carbamazepin-Derivates (Oxacarbazepin)

und eines Somatostatin-Analogs (SMS 201-995) durch Schlafmuster, EEG-Analyse und Hormonprofil

P. Clarenbach, E. Del Pozo, M. A. Kammerer-Hoch, F. Ries und

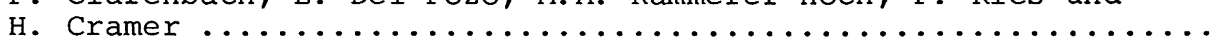

Das Bereitschaftspotential bei unilateraler chronischer Läsion der supplementär-motorischen Area (SMA)

W.J. Heller, W. Lang, M. Hufnagl, L. Deecke und H.H. Kornhuber . 891

Untersuchungen zur Frage des Kopfnystagmus beim Menschen

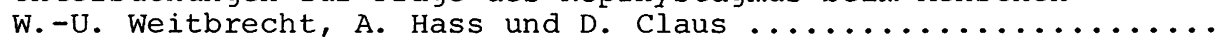

Möglichkeiten der computer-assistierten Signalerkennung in

der Elektromyographie zentralmotorischer störungen

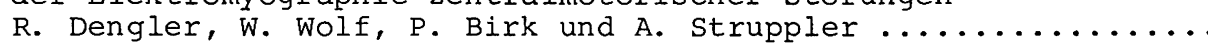

Horizontale Sakkaden bei Gesunden und Diabetikern - Ein Vergleich mit der Bestimmung der Nervenleitgeschwindigkeit

M. Hirschberg, M. Hesseling, I. Husstedt, B. Hofferberth und

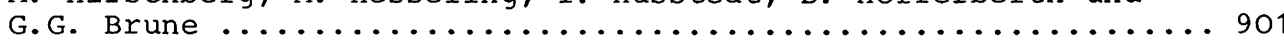

Störung der Exkretion von Hautoberflächenlipiden beim

Parkinsonsyndrom

H. Baas und P. -A. Fischer ........................... 905

Morbus Parkinson und Opioide. Bestimmung des Met-Enkephalin

und der biogenen Amine im Liquor cerebrospinalis und

Parkinson-antagonistischer Effekt vom Naloxon

H. Przuntek, K. Henning, H. Russ und H. Eckhardt ........... 909

Das Shy-Drager-Syndrom

W. Kohlhepp, H. Przuntek, R. Rohkamm und H. Wernze ......... 913

Zur Epidemiologie des Morbus Huntington in Unterfranken

B. Schrank, A. Steigerwald, J. Filger, W. Kuhn und H. Przuntek. 917

Blickkoordination bei Chorea Huntington: Klinische Befunde zur Kopf-Augen-Koordination

W. H. Zangemeister und A. Mueller-Jensen ................... 920

Familiäre paroxysmale dystone Choreathetose Mount-Reback:

Familienanamnese, Fallschilderung und Effekt von Clonazepam

A. Arlt und R.W.C. Janzen 
Vergleichende Untersuchungen des Gehirns von Patienten mit Morbus Wilson durch Computer- und magnetisch ResonanzfeldTomographie

K. Demisch, E. Schneider und D. Vonofakos .................

Klinische Beobachtung bei einer bioptisch gesicherten

juvenilen Form der neuro-axonalen Dystrophie

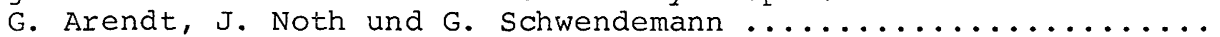

Neuropeptide bei degenerativen ZNS-Erkrankungen - Radioimmuno-

logischer Nachweis von Somatostatin und vasoaktivem intestinalem Polypeptid im Liquor cerebrospinalis

A. Weindl, J. Unger, H. Gnahn, H. Lange, W. Lange und

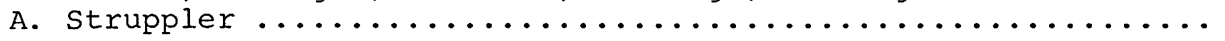

Posturographischer Nachweis von paraneoplastischer zerebellärer Degeneration bei Bronchialkarzinom

K. Wessel, H.C. Diener, H. Ackermann und E. Heidemann ........994

Die kortiko-nigrale Projektion benutzt Glutamat als Transmitter

J. Kornhuber, J.S. Kim, M.E. Kornhuber und H.H. Kornhuber ..... 946

Messung der Affinität von Dopamin-Rezeptoren im menschlichen Gehirn mittels 11C-Methyl-Spioerone und Positronen-EmissionsTomographie

S. Herold.

Erste Erfahrungen mit einer computer-gestützten Kopfschmerzdiagnostik

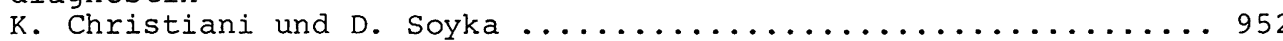

Migränesyndrom und Liquorpleozytose

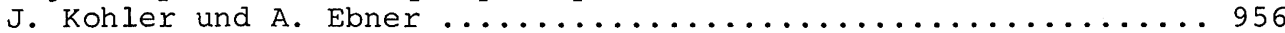

Therapeutische wirksamkeit eines psychophysiologischen

Trainingsprogramms bei Migränepatienten

M. Falkenstein, J. Zülch, J. Hoormann und E. Müller .........

Operationsergebnisse bei Gliomen in funktionell hochwertigen

Arealen der Großhirnhemisphären

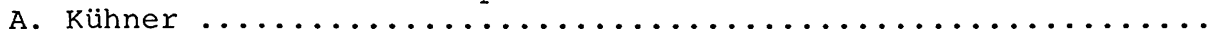

Spinales Trauma und Syringomyelie

B.C.G. Schalke, M. Ratzka, N. Sörensen und R. Rohkamm

Experimentelle Aspekte zur Immuntherapie in der Neuroonkologie: Induktion einer effektiven autologen zytotoxischen Reaktion bei malignen Hirntumoren

U. Bogdahn, L.B. Epstein, H.T.R. Rupniak und B. Fleischer .....

Immunzytochemische Untersuchungen zur Blut-Hirn-SchrankenStörung menschlicher Gliome

R.J. Seitz und $w$. Wechsler $\ldots \ldots \ldots \ldots \ldots \ldots \ldots \ldots \ldots \ldots \ldots \ldots \ldots . \ldots \ldots$

Erhöhte Vasopressinkonzentration im Liquor bei Pseudotumor cerebri

K. Wessel, H. Wiethölter und $w$. Knepel $\ldots \ldots \ldots \ldots \ldots \ldots \ldots$

Die wirkung von hypotensiv wirkenden Substanzen auf die regionale Hirndurchblutung und den Hirndruck von Pavianen mit normalem und erhöhtem intrakraniellen Druck

A. Hartmann, C. Buttinger und $\mathrm{Z}$. Czernicki 
Veränderungen der Serumosmolalität bei der Hirndruckbehandlung mit oraler und intravenöser Glyzerinabgabe

P.W. Schönle, S. Menck, H. Prange und E. Bollensen .......... 991

Psychophysiologische Untersuchungen an Aphasikern unter verschiedenen sprachlichen Anforderungen

H. Hielscher, H. Wilhem und R. Tigges ..................... 995

Ein epidemiologischer Beweis für die Wirksamkeit der Früh-

behandlung zerebraler Sprachstörungen im Kindesalter mit

Hilfe der Mütter

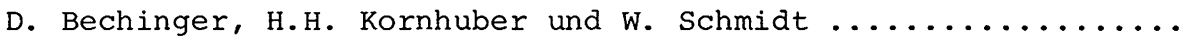

Schlucksynkopen als seltene Ursache nicht-epileptischer Anfälle J. Kotzian, K.-F. Druschky, H. Daun und R. Leutschaft ....... 1003

Der Effekt der Gewichtsreduktion auf Schlaf-Apnoe und

Hypersomnie beim Pickwick-Syndrom

C. Mohs, L. Lachenmayer, S. Zschocke und $\mathrm{K}$. Winckler ........ 1008

Die magnetische Resonanz Tomographie (MRT) in der Neurologie

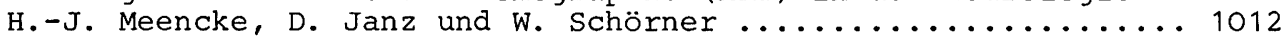

Kostenverminderung der Antikonvulsivabestimmung im homogenen

Enzym-Immunoassay (EMIT) im Vergleich zum Fluoreszenz-Polarisations-Immunoassay (FPIA)

T.O. Kleine, N. Arnold, H. Adam und H. Pick-Kober ......... 1017

Vergleichende Hirndurchblutungsmessung mit der intraarteriellen

133Xenon-Technik und der Mikrosphärenmethode bei Pavianen mit

fokaler transitorischer Hirnischämie

A. Hartmann, Z. Czernicki, S.-N. Reske, F.-J. Schuier,

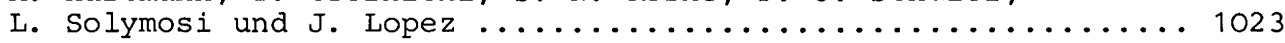

Eine nozifensive Reaktion der Katze und ihre Eignung zur schonenden Algesimetrie - Eine Verlaufsstudie über 120 Tage

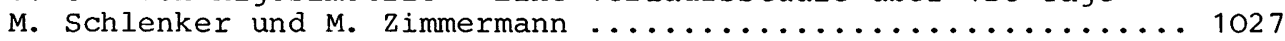

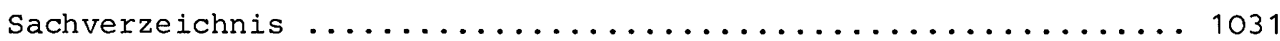




\title{
Zur Neurotoxizität von Antiepileptika bei Langzeitbehandlung
}

\author{
K.-H. Krause, P. Berlit und G. Kynast
}

Einleitung

Durch die Einnahme von Antiepileptika bedingte Schädigungen des zentralen und peripheren Nervensystems fanden in den letzten Jahren zunehmend Interesse. Am längsten bekannt sind wohl die bei toxischen Medikamentenspiegeln auftretenden Enzephalopathien, insbesondere die Kleinhirnschädigung. EEG-Veränderungen sind in Abhängigkeit vom Plasmaspiegel der Medikamente beschrieben (3). Medikamenteninduzierte psychotische Episoden, "alternative Psychosen", wurden erstmals von Landolt dokumentiert (14). Unter den neuropsychologischen Störungen fanden besonders sedative Effekte und Konzentrationsstörungen Beachtung. Im Bereich des peripheren Nervensystems sind Antiepileptikainduzierte Polyneuropathien beschrieben. Mit den beiden letztgenannten Formen der Neurotoxizität von Antiepileptika haben wir uns im Rahmen einer repräsentativen Untersuchung $z u$ Nebenwirkungen von Antiepileptika bei langzeitbehandelten Patienten in der Heidelberger Anfallambulanz näher befaßt.

Nervenleitgeschwindigkeit und Antiepileptika

Eine Beeinträchtigung von Funktionen des peripheren Nervensystems durch Phenytoin wird schon seit langem diskutiert (9). Gesichert ist, daß im Rahmen einer akuten Phenytoin-Intoxikation eine reversible Verlangsamung der Leitgeschwindigkeit peripherer Nerven nachweisbar ist $(1,12,17,19)$. Kontrovers ist die Diskussion hinsichtlich einer durch Phenytoin induzierten Polyneuropathie bei Langzeiteinnahme; bisher durchgeführte Studien kamen zu unterschiedlichen Resultaten, die bei einer Häufigkeit elektrophysiologisch faßbarer Anomalien zwischen $0 \%$ und $89 \%$ liegen $(2,4-8,10,15,21,22)$. Die Patienten mit chronischer Phenytoineinnahme wurden meist kombiniert mit weiteren Antiepileptika behandelt. Inwieweit diese anderen Antiepileptika einen möglichen Einfluß auf die Leitfunktion peripherer Nerven haben, ist $z . Z t$. offen; die Ergebnisse hinsichtlich der wirkung von Carbamazepin sind widersprüchlich $(19,21)$. Für die Monotherapie mit Barbiturat wurde neuerdings ein Einfluß auf die elektrophysiologischen Funktionen vermutet (19), was sich allerdings nur auf 6 monotherapierte Patienten stützt. Unklar sind die möglichen Beziehungen zwischen neurophysiologischen Parametern und durchschnittlicher Medikamentendosis sowie insgesamt zugeführter Medikamentenmenge bei langzeitbehandelten Epileptikern.

Mit Unterstützung durch die Deutsche Forschungsgemeinschaft ( $\mathrm{Kr}$ 659/1) 
Patienten und Methodik

Im Rahmen der Heidelberger Antiepileptikastudie zur chronischen Toxizität von Antikonvulsiva konnten von 610 20-40jährigen, mindestens ein Jahr lang mit Antiepileptika behandelten Patienten 580 neurophysiologisch untersucht werden. Nachdem 32 patienten mit möglichen anderen Ursachen einer Polyneuropathie (z.B. Diabetes mellitus, Niereninsuffizienz, Alkoholabusus) ausgeschlossen worden waren, verblieben 313 Männer und 235 Frauen, deren neurophysiologische Daten ausgewertet wurden. Da es sich meist um unter einer Kombinationstherapie stehende Patienten handelte, wurde die insgesamt eingenommene Antiepileptikamenge in Äquivalenzeinheiten berechnet ${ }^{1}$. Entsprechend der jeweiligen Behandlungsdauer wurde die durchschnittlich pro Tag eingenommene Antiepileptikamenge in Äquivalenzeinheiten pro Tag angegeben. 37 Patienten hatten bis zum Zeitpunkt der Untersuchung ausschließlich Phenytoin, 46 Primidon, 19 Carbamazepin und 13 Valproat eingenommen. Die Messung der Nervenleitgeschwindigkeiten erfolgte mit einem Disa-System 1500 bei konstanter Hauttemperatur von $34^{\circ} \mathrm{C}$. Die motorische Leitgeschwindigkeit des Nervus medianus rechts sowie des Nervus peronaeus rechts wurde mit Oberflächenelektroden bestimmt, die sensible Leitgeschwindigkeit des Nervus medianus rechts mit antidromer Technik (Ableitung der Antwortpotentiale mit Ringelektroden vom Mittelfinger). Sämtliche Patienten wurden gezielt nach dem Vorliegen subjektiver Beschwerden wie Kribbeln in den Extremitäten, brennenden Schmerzen oder Pelzigkeitsgefühl befragt; bei allen Patienten wurde der Reflexstatus an den unteren Extremitäten erhoben. Die Plasmaspiegel der Antiepileptika wurden teils gaschromatographisch, teils mit Radioimmunassay bestimmt.

Die Beziehungen zwischen neurographischen Parametern und Gesamt- bzw. durchschnittlicher Tagesdosis in Äquivalenzeinheiten wurden mit dem Verfahren der linearen Regression überprüft, ebenso mögliche Korrelationen zwischen Plasmaspiegel der Antiepileptika und Nervenleitgeschwindigkeiten. Die Werte der Leitgeschwindigkeiten bei den mit Phenytoin, Primidon, Carbamazepin oder Valproat monotherapierten Patienten wurden mit Hilfe der Varianzanalyse verglichen.

\section{Ergebnisse}

Die Mittelwerte \pm S.D. betrugen bei den ausgewerteten 548 Patienten für die motorische Leitgeschwindigkeit des $\mathrm{N}$. medianus am Unterarm $55,8 \mathrm{~m} / \mathrm{s} \pm 4,0 \mathrm{~m} / \mathrm{s}$ (Männer [n=313] $55,7 \mathrm{~m} / \mathrm{s} \pm 3,9 \mathrm{~m} / \mathrm{s}$, Frauen [n=235] $56,0 \mathrm{~m} / \mathrm{s} \pm 4,1 \mathrm{~m} / \mathrm{s})$, für die sensible Leitgeschwindigkeit des $\mathrm{N}$. medianus $61,1 \mathrm{~m} / \mathrm{s} \pm 4,8 \mathrm{~m} / \mathrm{s}$ (Männer $60,8 \mathrm{~m} / \mathrm{s} \pm 4,8 \mathrm{~m} / \mathrm{s}$, Frauen $61,4 \mathrm{~m} / \mathrm{s} \pm 4,8$ $\mathrm{m} / \mathrm{s})$ und für die Leitgeschwindigkeit des $\mathrm{N}$. peronaeus im Unterschenkelbereich $49,3 \mathrm{~m} / \mathrm{s} \pm 3,9 \mathrm{~m} / \mathrm{s}$ (Männer $48,7 \mathrm{~m} / \mathrm{s} \pm 3,9 \mathrm{~m} / \mathrm{s}$, Frauen 50,0 $\mathrm{m} / \mathrm{s} \pm 3,7 \mathrm{~m} / \mathrm{s})$. Alle drei bestimmten Leitgeschwindigkeiten korrelierten signifikant negativ mit den insgesamt zugeführten Antiepileptika in Äquivalenzeinheiten (Spearmanscher Rangkorrelationskoeffizient $r=$ $-0,125$ ( $\mathrm{N}$. medianus motorisch), $p=0,003 ; r=-0,158$ ( $\mathrm{N}$. medianus sensibel), $p=0,0002 ; r=-0,166$ ( $N$. peronaeus), $p=0,0001$ sowie mit der durchschnittlich pro Tag eingenommenen Antiepileptikamenge in Äquiva-

1 Dabei entsprechen einer Äquivalenzeinheit $50 \mathrm{mg}$ Phenytoin, $30 \mathrm{mg}$ Phenobarbital (= $50 \mathrm{mg}$ Barbexaclon), $125 \mathrm{mg}$ Primidon, $200 \mathrm{mg}$ Carbamazepin, $250 \mathrm{mg}$ Ethosuximid, $300 \mathrm{mg}$ Valproat, $50 \mathrm{mg}$ Mesantoin, $2 \mathrm{mg}$ Clonazepam, $300 \mathrm{mg}$ Mesuximid, $100 \mathrm{mg}$ Sulthiam und $250 \mathrm{mg}$ oxazolidin 
lenzeinheiten pro $\operatorname{Tag}(r=-0,100$ (N. medianus motorisch), $p=0,02$; $r=-0,141$ ( $N$. medianus sensibel), $p=0,001 ; r=-0,133$ (N. peronaeus), $\mathrm{p}=0,002$. Von den 548 patienten boten 21 eine motorische Leitgeschwindigkeit des $\mathrm{N}$. medianus unter $50 \mathrm{~m} / \mathrm{s}, 44$ eine sensible Leitgeschwindigkeit des $\mathrm{N}$. medianus unter $55 \mathrm{~m} / \mathrm{s}$ und 69 eine motorische Leitgeschwindigkeit des $\mathrm{N}$. peronaeus unter $45 \mathrm{~m} / \mathrm{s}$ (hierbei handelt es sich jeweils um die unteren Werte des Streubereiches der einzelnen Leitgeschwindigkeitsmessungen bei Normalpersonen in unserem EMG-Labor). Unter Berücksichtigung der überschneidungen innerhalb der einzelnen Gruppen verblieben 102 Patienten (= 19\% des ausgewerteten Kollektivs), die neurographisch auffällig waren. Von diesen 102 Patienten gaben 30 anamnestisch sensible Störungen an, drei davon in Kombination mit Reflexausfällen an den unteren Extremitäten; weitere drei Patienten aus dem Kollektiv mit erniedrigten Leitgeschwindigkeiten boten Reflexausfälle, berichteten aber nicht iber sensible störungen. Insgesamt hatten 49 patienten über Einschlaf- und Pelzigkeitsgefühl in den Extremitäten geklagt, 46 über Kribbeln und zwei über brennende Schmerzen; neurographisch auffällig mit mindestens einer verlangsamten Leitgeschwindigkeit waren hiervon jeweils 17, 21 und 2 Patienten. Von insgesamt 5 patienten mit beidseits nicht auslösbarem Quadrizeps-femorisReflex waren 3, von 10 Patienten mit beidseits fehlendem Tricepssurae-Reflex 4 neurographisch auffällig. Die bei den monotherapierten Patienten erhobenen neurographischen Parameter sind Tabelle $1 \mathrm{zu}$ entnehmen. Die bei Carbamazepin bestehende Tendenz zu niedrigeren Werten bei den Leitgeschwindigkeiten des $N$. medianus ließ sich statistisch nicht sichern. Weiterhin konnte eine negative Korrelation zwischen Leitgeschwindigkeit und Plasmaspiegel bei keinem der vier Antiepileptika festgestellt werden.

\section{Diskussion}

Die Häufigkeit von $19 \%$ abnormer neurographischer Befunde in unserem Krankengut entspricht weitgehend der von Lovelace und Horwitz (15) und Swift et al. (21) mitgeteilten Inzidenz. Bemerkenswert ist, daß weniger als ein Drittel unserer neurophysiologisch auffälligen Patienten zusätzlich anamnestisch oder klinisch Hinweise auf eine Polyneuropathie bot. Auf der anderen seite muß hervorgehoben werden, daß jeweils nur etwa die Hälfte der anamnestisch oder klinisch auffälligen Patienten auch erniedrigte Leitgeschwindigkeiten hatte. Möglicherweise wären mit zusätzlichen Messungen, etwa der Leitgeschwindigkeit des Nervus suralis, des H-Reflexes oder der F-Wellen, noch weitere Patienten als neurophysiologisch auffällig erfaßt worden; allerdings fanden Swift et al. (21), die entsprechende Untersuchungen bei ihrem Kollektiv durchführten, die höchste Inzidenzrate bei der Verlangsamung der Leitgeschwindigkeit des Nervus peronaeus.

Die gefundenen negativen Korrelationen sprechen prinzipiell dafür, daß zwischen Antiepileptikazufuhr und Leitfunktion der peripheren Nerven bei chronisch behandelten, nicht intoxikierten patienten ein Zusammenhang besteht; über welche Mechanismen dies erfolgt, ist unklar. Interessant dürfte zunächst sein, möglichen Verbindungen zum Vitaminstatus nachzugehen. Nach unseren Ergebnissen scheint der insgesamt zugeführten Medikamentenmenge - und somit der Behandlungsdauer eine noch wichtigere Rolle zuzukommen als der durchschnittlich pro Tag eingenommenen. Hierfür spricht auch, daß sich bei den monotherapierten Patienten keine Korrelation $z$ wischen neurographischen Parametern und Plasmaspiegeln fand. Unsere Ergebnisse bei den monotherapierten Patienten stützen im übrigen ganz eindeutig die von Swift et al. (20) geäußerte Ansicht, daß dem Phenytoin im Vergleich zu den anderen Anti- 
Tabelle 1. Werte ( $\bar{x} \pm$ s.d.) für Behandlungsdauer, mittlere Tagesdosis, Plasmaspiegel (PB = Phenobarbital), Nervenleitgeschwindigkeiten und Ergebnisse im d2-Aufmerksamkeitsbelastungstest ( $G Z=$ Ganzleistung, $G Z-F=$ Gesamttestwert) bei monotherapierten Patienten

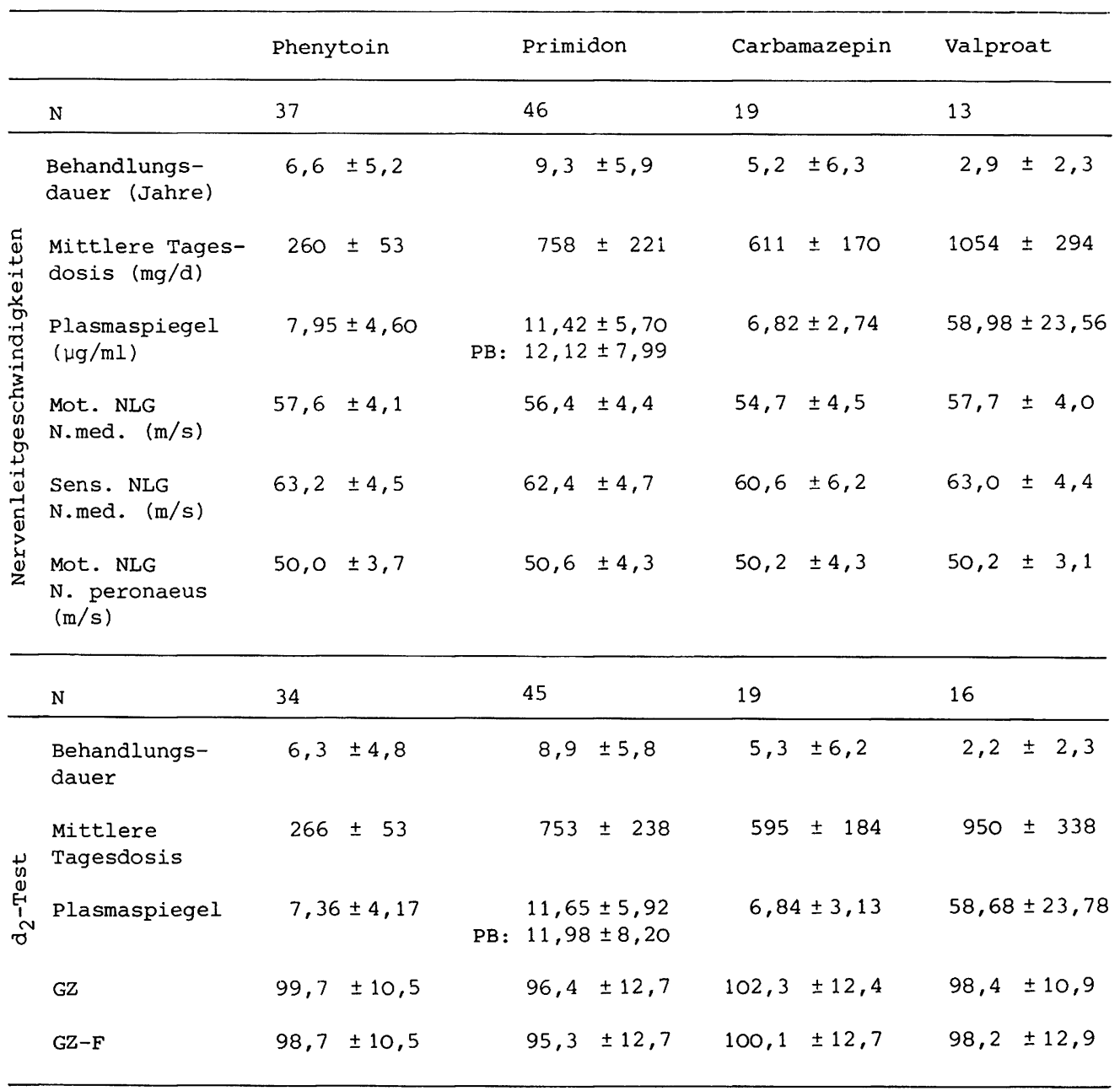

epileptika bei Langzeiteinnahme keinesfalls eine spezifische Beeinflussung der Leitfunktion peripherer Nerven anzulasten ist.

Konzentrationsfähigkeit und Antiepileptika

Zur Erfassung der Konzentrationsfähigkeit führte Remschmidt (18) bei 60 Epileptikern, 30 Gesunden und 20 internistisch Kranken den $d_{2}$-Aufmerksamkeits-Belastungstest durch; er fand hierbei eine deutlich schlechtere Leistung bei den Epileptikern, wobei möglichen Zusammenhängen mit der Medikation nicht nachgegangen wurde. Zehn Jahre später untersuchten Marchesi et al. (16) diesen Aspekt; die Autoren fanden 
für die mit Phenytoin und Phenobarbital behandelten Patienten eine Verschlechterung der Aufmerksamkeitsleistung, für die mit Carbamazepin therapierten war kein Einfluß feststellbar. Eine größere Anzahl von Epileptikern wurde von Hebenstreit (11) mit Hilfe des $d_{2}$-Tests untersucht; bei den ambulanten Epileptikern ergab sich hierbei keine Abweichung von der Testnorm - im Gegensatz zu den stationären Patienten; Unterschiede zwischen den einzelnen Epilepsieformen wurden nicht festgestellt, Beziehungen zur Medikation nicht überprüft. Hunger und Kleim (13) beschrieben im Gegensatz hierzu kürzlich Leistungsdefizite beim $\mathrm{d}_{2}$-Test vor allem bei Patienten mit diffusem generalisiertem Anfallstyp. In der vorliegenden Studie sollen folgende nach der Literatur noch offene Fragen untersucht werden:

1. Gibt es einen Unterschied zwischen Patienten mit idiopathischer und symptomatischer Epilepsie in der Konzentrationsfähigkeit?

2 . Besteht ein Zusammenhang zwischen Konzentrationsfähigkeit und antiepileptischer Medikation?

3. Finden sich Unterschiede im $\mathrm{d}_{2}$-Test zwischen den monotherapierten Patienten?

\section{Patienten und Methodik}

Nach Ausschluß von Patienten mit schweren zerebralen Schäden, Hemiparese, Sehstörungen und dementer Entwicklung verblieben 505 20-40jährige Epileptiker (298 Männer, 207 Frauen), bei denen der d $d_{2}$-Aufmerksamkeits-Belastungstest nach Brickenkamp durchqeführt wurde. Bei 194 Patienten bestand eine symptomatische, bei 311 eine idiopathische Epilepsie. Die insgesamt und durchschnittlich eingenommene Dosis in Äquivalenzeinheiten wurde wie oben dargestellt errechnet. 34 Patienten waren bis zum Untersuchungszeitpunkt ausschließlich mit Phenytoin, 45 mit Primidon, 19 mit Carbamazepin und 16 mit Valproat behandelt worden. Statistisch wurde die Ganzleistung (Gesamtzahl = GZ) sowie der Gesamttestwert (Gesamtzahl - Fehler = GZ - F) mit den Medikamentenmengen korreliert (Verfahren der linearen Regression), die Werte für die idiopathische und symptomatische Epilepsie wurden mit dem t-Test, die Werte bei den Monotherapien mit Hilfe der Varianzanalyse verglichen. Mögliche Korrelationen zwischen Testergebnis und AntiepileptikaPlasmaspiegeln bei den Monotherapierten wurden überprüft.

\section{Ergebnisse}

GZ lag bei unserem Kollektiv durchschnittlich bei 95,9 $\pm 11,7$ (Männer 95, $0 \pm 11,2$, Frauen 97,2 $\pm 12,2$ ), GZ-F bei $94,8 \pm 11,9$ (Männer 94,2 $\pm 11,7$, Frauen 95,7 $\pm 12,2)$. Die Patienten mit idiopathischer Epilepsie hatten einen Wert von $97,0 \pm 11,5$ für GZ, die mit symptomatischer einen Wert von $94,2 \pm 11,7$ (Unterschied mit $\mathrm{p}=0,0104$ signifikant); für GZ-F sind die Werte $96,1 \pm 11,8$ bei den idiopathischen und $92,7 \pm 11,8$ bei den symptomatischen Epilepsien $(p=0,0015)$. Die Korrelation zwischen den Ergebnissen im $d_{2}$-Test und der Gesamt-bzw. Tagesdosis der Antiepileptika sind Tabelle 2 sowie den Abbildungen 1 und $2 \mathrm{zu}$ entnehmen. GZ und GZ-F bei den monotherapierten Patienten sind in Tabelle 1 wiedergegeben. Der Trend zu niedrigeren Werten bei Primidon ließ sich statistisch nicht sichern; ebenso fanden sich keine positiven Korrelationen zwischen Resultaten im $\mathrm{d}_{2}$-Test und den Plasmaspiegeln der Antiepileptika bei den vier Gruppen mit Monotherapie. 
Tabelle 2. Spearmanscher Rangkorrelationskoeffizient $r$ für die Beziehungen zwischen insgesamt ( $\ddot{E}$ ges.) bzw. durchschnittlich pro Tag ( $\ddot{A} E / d)$ eingenommenen Antiepileptika in Äquivalenzeinheiten und den Ergebnissen im $d_{2}$-Test beim Gesamtkollektiv sowie der Patientengruppe mit idiopathischer und symptomatischer Epilepsie; * nach der Bonferroni-Holm-Methode (20) signifikant zum Niveau 0,05

\begin{tabular}{|c|c|c|c|}
\hline & $\begin{array}{l}\text { Gesamt } \\
(n=505)\end{array}$ & $\begin{array}{l}\text { Idiopathische } \\
\text { Epilepsie } \\
(\mathrm{n}=311)\end{array}$ & $\begin{array}{l}\text { Symptomatische } \\
\text { Epilepsie } \\
(\mathrm{n}=194)\end{array}$ \\
\hline$\ddot{A E} / \mathrm{d}-\mathrm{GZ}$ & $-0,2205 \mathrm{p}=0,0001^{\star}$ & $-0,2760 \mathrm{p}=0,0001^{\star}$ & $-0,1239 p=0,09$ \\
\hline$\ddot{A} E$ ges. - GZ & $-0,2117 p=0,0001^{*}$ & $-0,2468 p=0,0001 *$ & $-0,1579 p=0,03$ \\
\hline$\ddot{A} E / d-G Z-F$ & $-0,2471 \mathrm{p}=0,0001 *$ & $-0,3044 p=0,0001 *$ & $-0,1487 p=0,04$ \\
\hline$\ddot{A E}$ ges. - GZ-F & $-0,2596 \mathrm{p}=0,0001 *$ & $-0,2876 \mathrm{p}=0,0001^{\star}$ & $-0,2168 \mathrm{p}=0,002^{\star}$ \\
\hline
\end{tabular}

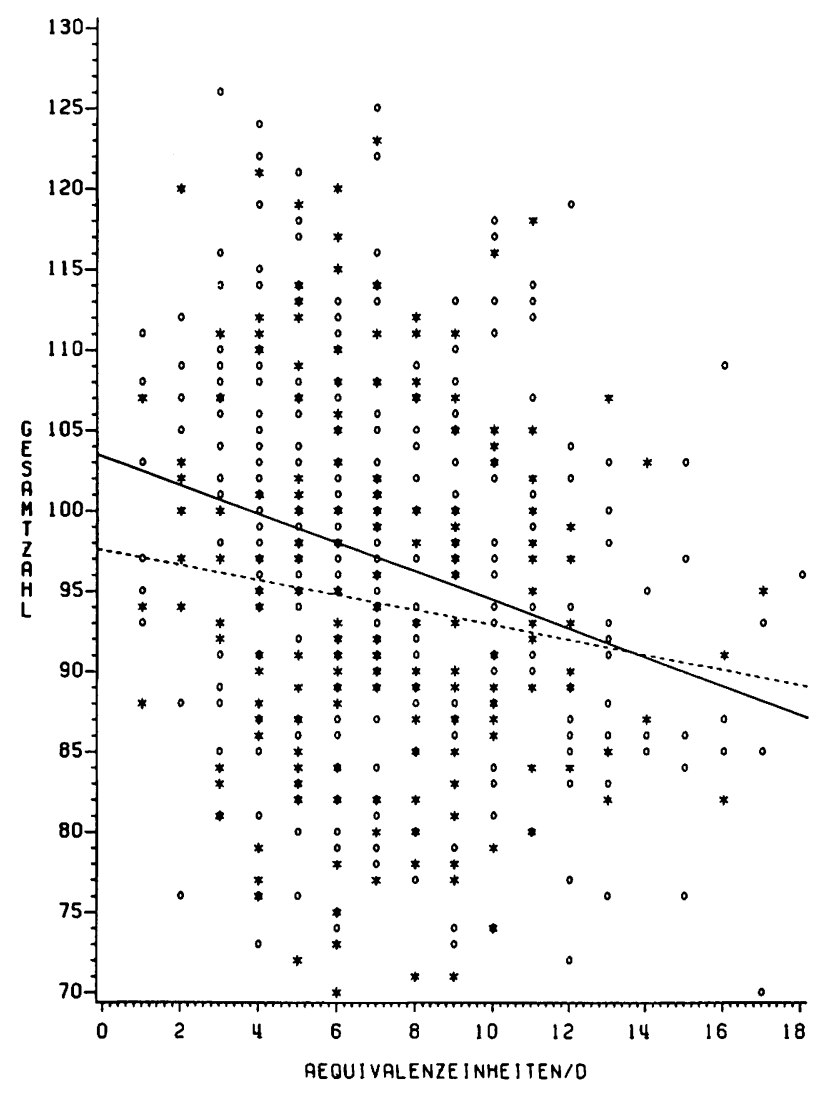

Abb. 1. Beziehung zwischen durchschnittlicher Antiepileptika-Tagesdosis in Äquivalenzeinheiten/ $d$ und im $\mathrm{d}_{2-}$ Aufmerksamkeitsbelastungstest erreichter Gesamtzahl bei Patienten mit idiopathischer (o, Regressionsgerade und symptomatischer ( $*$, Regressionsgerade ----) Epilepsie 


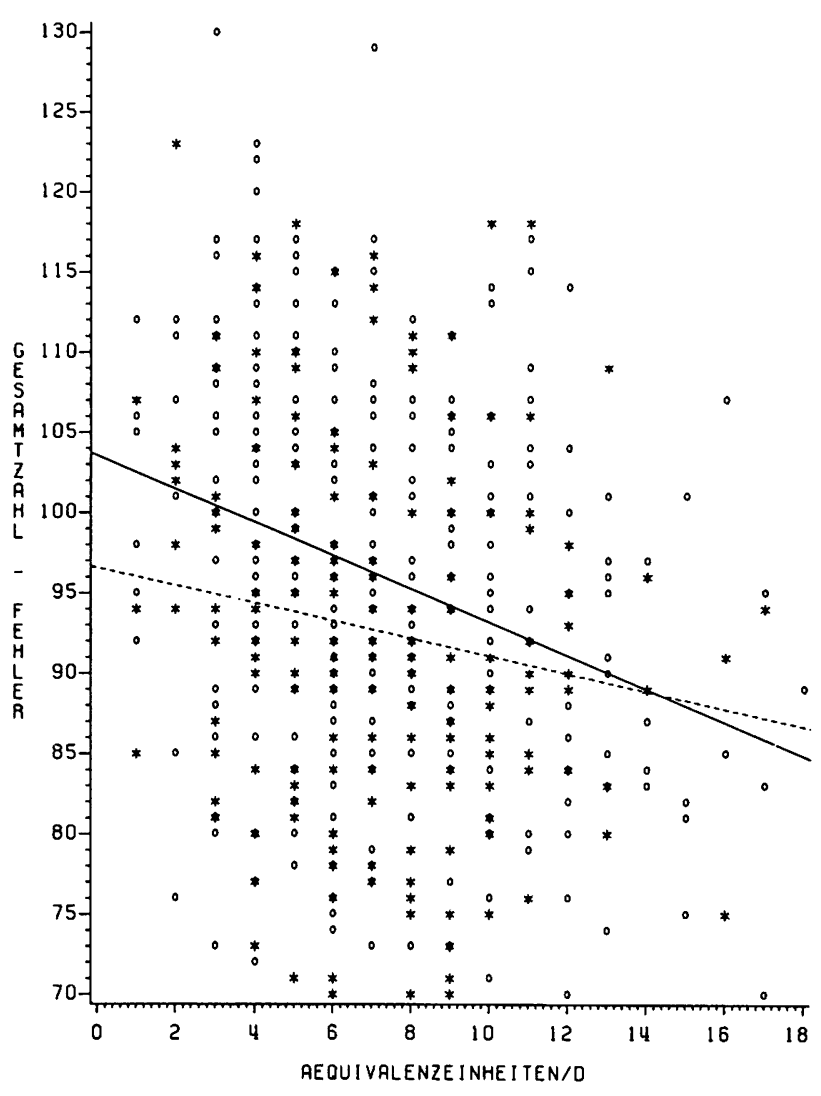

Abb. 2. Beziehung zwischen durchschnittlicher Antiepileptika-Tagesdosis und Gesamttestwert (Gesamtzahl - Fehler) im $\mathrm{d}_{2}$-Test (sonst wie Abb. 1)

\section{Diskussion}

Die von uns für das Gesamtkollektiv festgestellten erniedrigten Werte für die Konzentrationsleistung entsprechen weitgehend den von Hunger und Kleim (13) bei ihrem Epileptikerkollektiv gefundenen. Daß hierbei möglicherweise die Medikation ein mitverursachender Faktor ist, zeigen die signifikanten negativen Korrelationen zwischen Medikamentendosis und Leistung im $\mathrm{d}_{2}$-Test; es ist hierbei natürlich $\mathrm{zu}$ beachten, daß die Patienten mit den höchsten Dosierungen die schwersten Epilepsieformen haben und somit sicherlich von vornherein eine schlechtere Konzentrationsfähigkeit aufweisen. Erwartungsgemäß unterscheiden sich hinsichtlich der Konzentrationsfähigkeit die Patienten mit idiopathischer von denen mit symptomatischer Epilepsie. Die Tatsache, daß nur bei der ersten Gruppe sämtliche Korrelationen zu Gesamt- bzw. durchschnittlicher Tagesdosis signifikant waren, spricht dafür, daß bei diesem Epilepsietyp der Medikation hinsichtlich der Konzentrationsfähigkeit wohl eine erheblich größere Rolle zukommt als bei der symptomatischen Epilepsie, wo entsprechende Beziehungen nur teilweise nachweisbar waren. Hier führt offenbar häufig schon das Grundleiden selbst zum schlechteren Testergebnis ohne wesentliche Beeinflussung durch die Medikation. Diese Befunde stehen in einem gewissen Gegensatz zu den erwähnten Ergebnissen von Hebenstreit (11), der allerdings nicht Epilepsie-, sondern Anfallstypen verglich und hierbei keine Unterschiede fand. Von praktischer Wichtigkeit ist, daß bei Monotherapien mit Phenytoin, Carbamazepin und Valproat von den Patienten durchschnittlich 
normale Werte erreicht werden, während die Konzentrationsfähigkeit bei Primidon deutlich geringer ist und weitgehend der des Gesamtkollektivs gleicht. Das Ergebnis von Marchesi et al. (16) hinsichtlich eines negativen Effektes der Phenytoingabe auf die Aufmerksamkeitsleistung fanden wir an unserem Kollektiv nicht bestätigt.

\section{Zusammenfassung}

Von 548 elektroneurographisch untersuchten Epileptikern boten 19\% mindestens eine verminderte Nervenleitgeschwindigkeit. Es fanden sich signifikante negative Korrelationen zwischen Leitgeschwindigkeiten und insgesamt bzw. täglich zugeführter Medikamentenmenge. Die mit Phenytoin monotherapierten Patienten wiesen im Vergleich zu den mit anderen Antikonvulsiva behandelten keine spezielle Beeinträchtigung der Leitfunktion auf. Auch beim $d_{2}$-Aufmerksamkeits-Belastungstest ( 505 Patienten) bestanden signifikant negative Korrelationen zur Gesamtmenge und Tagesdosis der Antiepileptika. Die Patienten mit symptomatischer Epilepsie schnitten schlechter ab als die mit idiopathischer.

\section{Literatur}

1. Birket-Smith E, Krogh E (1971) Motor nerve conduction velocity during diphenylhydantoin intoxication. Acta Neurol Scand 47: 265-271

2. Chokroverty S, sayeed ZA (1975) Motor nerve conduction study in patients on diphenylhydantoin. J Neurol Neurosurg Psychiatry 38: 1235-1239

3. Danner $R$ (1983) Nebenwirkungen von Phenytoin und Carbamazepin auf elektrophysiologische Funktionen des peripheren und zentralen Nervensystems. Nervenarzt $54: 530-534$

4. Danner R, Partanen VJ, Riekkinen P (1981) Chronic anticonvulsive therapy, peripheral nerve conduction velocity and EMG. Epilepsia $22: 675-687$

5. De Castro JHX, Acosta ML, Sica REP, Guerico N (1972) Sensory and motor nerve conduction velocity in long-term diphenylhydantoin therapy. Arq Neuropsiquitr (Sao Paulo) 30:215-220

6. Dobkin BH (1977) Reversible subacute peripheral neuropathy induced by phenytoin. Arch Neurol 34:189-190

7. Eisen AA, Woods JF, Sherwin AL (1974) Peripheral nerve function in long-term therapy with diphenylhydantoin. Neurology 24:411-417

8. Encinoza $O$ (1974) Nerve conduction velocity in patients on longterm diphenylhydantoin therapy. Epilepsia 15:147-154

9. Finkelman J, Arieff AJ (1942) Untoward effects of phenytoin sodium in epilepsy. JAMA 118:1209-1212

10. Fujiwara T, Seko K, Akiguchi I, Yamada N, Iwai N (1979) Peripheral nerve function in patients with long-term anticonvulsant therapy. Clin Neurol (Jpn) 19:735-743

11. Hebenstreit G (1982) Psychopathologie der Aufwach- und Schlafepilepsie. Nervenarzt 53:287-290

12. Hopf HC (1968) Über die Veränderung der Leitfunktion peripherer motorischer Nervenfasern durch Diphenylhydantoin. Dtsch $\mathrm{Z}$ Nervenheilk 193:41-56

13. Hunger J, Kleim J (1983) Testpsychologische Leistungsprüfung bei Epileptikern. Arch Psychiatr Nervenkr 233:307-325

14. Landolt H (1956) L'électroencéphalographie dans les psychoses épileptiques et les épisodes schizophréniques. Rev Neurol 95:597-599 
15. Lovelace RE, Horwitz SJ (1968) Peripheral neuropathy in long-term diphenylhydantoin therapy. Arch Neurol 18:69-77

16. Marchesi GF, Ladavas E, Provinciali L, Del Pesce M, Fuà P, Giuliani G (1980) Neuropsychological performances in patients treated with different antiepileptic drugs. In: Majkowski J (ed) Epilepsy: A clinical and experimental research. Karger, Basel, p 258

17. Meienberg O, Bajc O (1975) Akute Polyneuropathie durch Diphenylhydantoin-Intoxikation. Dtsch Med Wochenschr 100:1532-1539

18. Remschmidt $\mathrm{H}$ (1970) Experimentelle Untersuchungen zur sogenannten epileptischen Wesensänderung. Fortschr Neurol Psychiat 38:524-540

19. Shorvon SD, Reynolds EH (1982) Anticonvulsant peripheral neuropathy: a clinical and electrophysiological study of patients on single drug treatment with phenytoin, carbamazepin or barbiturates. J Neurol Neurosurg Psychiatry 45:620-626

20. Sonnemann E (1982) Allgemeine Lösungen multipler Testproblematik. EDV Med Biol 13:120-128

21. Swift TR, Gross JA, Ward LC, Crout BO (1981) Peripheral neuropathy in epileptic patients. Neurology 31:826-831

22. Zebrowska-Szymusik M (1978) Effect of phenytoin on peripheral motor neurons. Neurol Neurochir Pol 12:427-434 


\section{Sachverzeichnis}

Aachener Aphasietest 724,995

Abducensparese 660

Acetylcholin-Rezeptor-Antikörper 851

Acetylsalizylsäure $252,254,302$

Aciclovir $750 \mathrm{ff}$.

Acrodermatitis atrophicans 747

Acrilamid 325

Adam-Stokes-Anfall 158,274

Adenylatkinase $147 \mathrm{ff}$.

Adipositas $228 \mathrm{ff} ., 252$

ADP 111

Adrenalin $647 \mathrm{ff} .914$

Adriblastin 942

Aethylenoxid 364

Aflatoxine 371

Afterload $29 \mathrm{f}$.

Agarose $799 \mathrm{ff}$.

Aids $753 \mathrm{ff}$.

Akinese 917

akinetischer Mutismus 186

Akrylamid $364 \mathrm{f}$.

Akrylphosphate 367

akustisch evozierte Potentiale

$88 \mathrm{ff} ., 183,446 \mathrm{ff}, 509 \mathrm{ff}$.

$547,598 \mathrm{ff} ., 613 \mathrm{ff} ., 637,649$, $667,679,729,932$

- bei Alkoholismus 509

- beim Hirntod 547,598 ff. $603 \mathrm{ff} .608 \mathrm{ff}$.

- und Intensivneurologie $613 \mathrm{ff.}$ 649

Albumin 232

Aldosteron 914

Algesimetrie $1027 \mathrm{ff}$.

Aliasing 63

Alkohol 154,196,228 ff.,297 ff., $442 \mathrm{ff}, 0446 \mathrm{ff} ., 455 \mathrm{ff} ., 854$, 925

-, Anfälle 443

-, chronischer Abusus $208 \mathrm{ff}$. , 442 ff.

-, Delirium tremens $443 \mathrm{f}$.

-, Embryopathie 230

-, EMG 455 ff.

-, evozierte Potentiale $446 \mathrm{ff}$.

-, Hirnatrophie 467
-, Hirnstammaffektion $446 \mathrm{ff}$.

-, Kleinhirnatrophie 444

-, Polyneuropathie 443

-, Tabak-Alkohol-Amblyopie $451 \mathrm{ff}$.

-, Wernicke Enzephalopathie 443,458 ff.

-, zentrale pontine Myelinolyse $463 \mathrm{ff}$.

Alkylnitrosoharnstoffe 371

Alkylphosphate 319

Alkyltransferase 374,377

Allergie 505

Alodezie $349 \mathrm{f} ., 363,380,392,413$

Alpha-Koma 90

Alpha-2-Makroglobulin 232

Alpharezeptorenblocker $39 \mathrm{f}$.

Amantadin 213

Amaurosis 235

Amaurosis fugax 215

- bei Lues 239

Aminoglykoside 14,601

Amnesie 188

-, amnestisches Syndrom 192

-, transiente globale 193

Amöbiasis $516 \mathrm{f}$.

AMP, zyklisches $180 \mathrm{f}$.

Amphetamin 469

Amplitudenschwankungen 55

amyotrophe Lateralsklerose 797, $828,837,841 \mathrm{ff} .935$

analoge intravenöse subtraktionsangiographie (ASA) $134 \mathrm{ff}$.

Analog-Intensität-Frequenzregistrierung 55

Analogsignal 54

Anämie

-, Eisenmangel 249

-, hypochrome 237

-, normochrome 237

anaphylaktoide Reaktion 301

Anästhesia dolorosa $417 \mathrm{ff}$.

Aneurysma $13 \mathrm{ff}, 36 \mathrm{fo,256} \mathrm{ff}$.

-, disseziierendes 84

-, Ruptur 259

-, Ruptur eines Aortenaneurysmas 221 
Aneurysma

- im Ventrikel 50

Anfall

- bei Alkoholismus 443,448

- , kardiovaskulärer 198

-, psychomotorischer 198

- bei Thymoleptikaintoxikation 525

Angiitis

-, granulomatöse 247 ff.

Angina pectoris $34 \mathrm{f}$.

Angiofibrom 192

Angiographie, zerebrale $5 \mathrm{f}$, $17,49,63,67 \mathrm{f} ., 79,88,108,133 \mathrm{ff}$,

$143 \mathrm{f} ., 154 \mathrm{f},, 167 \mathrm{ff},, 243,249$, $304,306,537 \mathrm{f} ., 556 \mathrm{f} ., 627 \mathrm{f} .$, 673

- ASA 133 ff.

- Basilaristhrombose 587

-, Befunde bei Hirnembolie 154 ff.

- DSA 133 ff.

- beim Hirntod 537 f.,544,556 f., $562,603,621,627$

- ISA $133 \mathrm{ff.}$

- Komplikationen bei 193,221

- bei Lindau-Tumor 271

- bei Lues 240

-, Radionuklide 621

- transfemorale 105

-, Zeitpunkt bei Embolie 155

Angiokardiographie 46,108

Angiokardiopathie, angeborene $44 \mathrm{ff}$.

Angiom 264

Angiomatosis retinae 272

Angiopathie

- luetische $239 \mathrm{ff}$.

Angioplastie, perkutane transluminale $309 \mathrm{ff}$.

Anhidrosis $363,365,913$

Antepan $400841 \mathrm{ff}$.

Anthropologie 566

Antiarrhythmika 10

Antibiotika $505 \mathrm{ff}$.

-, intrathekale Gabe 505 ff.

Antidepressiva, trizyklische

- und Herz 11

Antielastintiter 165

Antiepileptika $471 \mathrm{ff}, 480 \mathrm{ff}$.

- Bestimmung $1017 \mathrm{ff}$.

Antigen $789,803 \mathrm{ff}$.

Antikoagulantien $5,10,16,22,60$, $62,64,108,156,250,264,267$

-, Endokarditis 16

-, Kardiomyopathie 22

Antikonvulsiva, s. Antiepilepti$\mathrm{ka}$

Antikörper $683 \mathrm{ff}, 687 \mathrm{ff}$, $788 \mathrm{ff.}, 803 \mathrm{ff}$.

-, antinukleäre 254
- Leu $7788 \mathrm{ff}$.

-, monoklonale $703,708 \mathrm{ff}$, $788 \mathrm{ff.,803} \mathrm{f}$.

- , Organ 254

Antilymphozytenserum 570,574

Antithrombin-3-Mangel $164 \mathrm{f}$.

Anzapfsyndrom 309

Aortenaneurysma 36

Aortenisthmusstenose $44 \mathrm{ff}$.

Aortenklappe $12 \mathrm{f}$.

-, Insuffizienz 250

-, Verkalkung 50

Aortensegel 250

Aortenstenose 29,50

Aortenvitium 103

-, kombiniertes $50 \mathrm{f}$.

apallisches Syndrom 9,11,590 ff.

Aphasie $34,144,153$ ff.,252, 995 ff.

- bei Hirnembolie 154

- beim lakunären Infarkt 144

-, thalamische 268

Apnoe 543,578 ff.

Approximation 93

Äquäduktverschluß 980

Ara-C 506

Arachidonsäure 826

Arachidonsäuremetabolit 259,826

Arachidonsäure-Thromboxan-Prostacyclin-system 259

Armplexus 295

Arrhythmie $9 \mathrm{ff}, 34 \mathrm{f}, 197$

-, absolute 294

- bei Friedreich-Ataxie 206

- respiratorische 197,208

- , ventrikuläre 276

Arsenvergiftung $361,403 \mathrm{ff}$.

Arteria basilaris

- Prognose bei Verschluß $177 \mathrm{ff}$.

-, Thrombose der 88,177 ff., $305,587 \mathrm{ff}$.

-, Verschluß 88,177 ff.,304, $587 \mathrm{ff}$.

Arteria carotis

- Chirurgie der $98 \mathrm{ff}$.

- Endarteriektomie 98

-, Stenose 125,192,216

-, Thrombose 103

-, Verschluß 120 f.,216,287

Arteria cerebelli inferior posterior 190

Arteria cerebri media 239,294, 301

Arteria cerebri posterior

-, Infarkt 294

- bei Lues 240

Arteria communicans posterior 195,304

Arteria interpeduncularis profunda 188 
Arteria ophthalmica 237

Arteria radialis 297

Arteria subclavia 309

Arteria temporalis 243,959

-, Biopsie der 235,243

Arteria vertebralis 304

-, Abgangsstenose $309 \mathrm{ff}$.

Arteriae lenticulostriatae $305 \mathrm{f}$.

Arteritis bei Lues 240

Arteriitis temporalis $235 \mathrm{ff}$.

arterioarterielle Embolie 155

Arteriografie 253

Arteriosklerose $167 \mathrm{ff},, 212,277$, 301

- der Rückenmarksgefäße 221

arteriosklerotische Frühveränderung 79

Aspartat 946

Asphyxie 554

Astrozytom $963,971,976$

Asystolie $274,276,281,283,287 \mathrm{ff}$.

Ataxie $247,271,305,481,489,678$, $729,737,913,941$

Ateminsuffizienz 280

Atemmittellage 206

Atemregulationsstörung, zentrale 250

Atemstillstand 539,543

Atropin $208 \mathrm{f} ., 280,527,596$

Atropintest $578 \mathrm{f}$.

Audiosignal-Analyse $54 \mathrm{f}, 58$

auditorisches system $480 \mathrm{ff}$.

Augenhintergrund 17

-, Endokarditis 17

Australia-Antigen 254

Auswurffraktion $27 \mathrm{f}$.

Automatismen, orale 247

autonomes Nervensystem $361 \mathrm{ff}$.

Autonomie, spinale 222

Autoregulation 277

AV-Blockierung $9,274,284$

Avidin 820

Azathioprin $254,570,574,683 \mathrm{ff}$. , $691,715 \mathrm{ff}$.

Azetylcholinesterase 319

Azoverbindungen 372

Baclofen 925

BAL-Dimercaprol 362,405

Ballonatrioseptostomie 46

Ballondilation 309

Bandpässe 55

Bannwarth-Syndrom $744 \mathrm{ff}$.

Barbiturate $181 \mathrm{ff} .469,475,557$, $580 \mathrm{f} ., 615,639$

- und Hirntod $580,615,648 \mathrm{f}$.

Bartlett-Methode 881

Basalganglien-Infarkt 145

Basilarisknopf 60

Basilarisverschluß 634,674

B-Bild $60,66,68,79$ ff.
BCNU 492

Beatmung $594 \mathrm{f}$.

Beckenkammbiopsie 254

Bemegrid-Provokation $197 \mathrm{f}$.

benigne monoklonale Gammopathie $784 \mathrm{f}$.

Benzodiazepine 469

Benzol 366

Beobachtungszeit $544 \mathrm{f}$.

BERA $482 \mathrm{f}$.

Bereitschaftspotential $878 \mathrm{ff}$, $891 \mathrm{ff}$.

Berliner Blau $383 \mathrm{ff} ., 392$

Bernoulli-Gesetz 67

Berufskrankheit $318,325 \mathrm{ff}$, $337 \mathrm{f} ., 340 \mathrm{ff} ., 389 \mathrm{ff},, 412$

- , Arzte 318

-, Braunsteinminen 412

-, Zementarbeiter $389 \mathrm{ff}$.

Beschallung, transorbitale 61

Beta-2-Mikroglobulin-Clearance 513

Betarezeptorenblocker 22,32 ff., $46,110,254,595$

Beta-Thromboglobulin $105 \mathrm{ff}$. 165,254

Beugeschablonen 585

Bewegungsstörung, zentrale $897 \mathrm{ff}$.

Bewußtseinsstörung $9 \mathrm{f}, 15,34$, $153 \mathrm{ff},, 173 \mathrm{ff} ., 463 \mathrm{f} ., 525 \mathrm{f}$.

- bei kardialer Hirnembolie $153 \mathrm{f}$.

- bei Thymoleptikaintoxikation $525 \mathrm{f}$.

- bei zentraler pontiner Myelinolyse 464

Bifurkation 81

Bing-Horton-Syndrom 953

biogene Amine 910

Bioprothese 16

Biopsie 164

Bioreceptor $322 \mathrm{f}$.

Biotin $412,820 \mathrm{ff}$.

Biphenyle $366 \mathrm{f}$.

Blalock-Taussig-Anastomose 45

Blasenstörung 222,969

Bleivergiftung $317 \mathrm{f} ., 325 \mathrm{f}$, $361 \mathrm{f} ., 395 \mathrm{ff}$.

Blepharospasmus 662

Blickkoordination bei Chorea Huntington $920 \mathrm{ff}$

Blickparese $186 \mathrm{ff}$.

-, vertikale $186 \mathrm{ff}$.

Blinkreflex $608 \mathrm{ff}, 637$

Blutbild 254

Blutgasanalyse 204

Blut-Hirn-Schranke $492,500,751$, $820,855,957$

- bei Gliomen 976 ff.

- bei Lues 240 
Bluthochdruck, s. Hypertonus

Blut-Liquor-Schranke $232 \mathrm{ff}$., 983

-, Störung der 232,846

Blut-Nerv-Schranke 820

Blutsenkung 237,249,254

-, Erhöhung der 237,247

Blutungsneigung 487

Blutzucker 254

Botulismus $318,424 \mathrm{ff}$.

Bradykardie $50,159,192,256,274$, 277,290

- bei Hirninfarkt 10

Bromocriptin 213,888

Bronchialkarzinom $941 \mathrm{ff}$

Bronchopneunomie 295

Budipin $420 \mathrm{ff}$.

Bulbusdruckversuch $280 \mathrm{ff}$.

"burst"-Aktivität $737 \mathrm{ff}$.

Butan 333 f.,365

B-Vitamine 452

Bypass-Operation 70

-, aortokoronare 72,294 ff.

-, Externa-Interna 244

Calciumantagonisten $39 \mathrm{f}$.

Ca-Na-EDTA $361,397,415$

Cannabis 469

Capsula interna 267

Carbamazepin $472 \mathrm{ff}, 480 \mathrm{ff}$. 851,886 ff. , 925, 1017

CBE/CBV-Quotient $124,127 \mathrm{f}$. , $129 \mathrm{ff}$.

Cerebral Blood Flow (CBF) $119 \mathrm{ff}, 124 \mathrm{ff} ., 127 \mathrm{f.,1} 129 \mathrm{ff}$.

Cerebral Blood Volume (CBV) $124 \mathrm{f.}, 127 \mathrm{f.,} 129 \mathrm{ff}$.

Chelatbildner 361,405

Chinidin $10 \mathrm{f}$.

Chlomethiazol $297 \mathrm{ff}$.

Chlorakne $319 \mathrm{f}$.

Cholinacetyltransferase 937

Chorea Huntington 92,897,917 ff., 920 ff.,932,935

Choree fibrillaire de Morvan $851 \mathrm{ff}$.

Choreoathetose Mount-Reback 924 ff.

Churg-Strauss 244

Circulus arteriosus Willisi $60 \mathrm{ff}$.

Cis-Platin $496 \mathrm{ff}$.

Clonazepam $489 \mathrm{ff.}, 924 \mathrm{ff}$.

Clonidin 39

C-Methyl-Spiperone $949 \mathrm{ff}$.

$\mathrm{CO}_{2}$-Atemantwortkurve $546 \mathrm{f}$.

Coeruloplasmin $928 \mathrm{ff}$.

Colliculus inferior $88 \mathrm{f}$.

Coma vigile 89,618

Computertomografie $16,60,88$,
142 ff.,155,180 ff.,187,190,

$192 \mathrm{f.}, 196,232 \mathrm{ff},, 243,250$,

$252 \mathrm{f} ., 294,305,343,431,460 \mathrm{f}$. ,

$627 \mathrm{ff} ., 634 \mathrm{ff} ., 673,679,729$,

$750,754,763,846,891,914,956$, 1005

- bei Dystrophia myotonica $833 \mathrm{ff}$.

- bei der Hirnembolie 155

- beim Hirninfarkt 142 f.,147

- beim Hirntod 538 f.,550,591 f., $627 \mathrm{ff} ., 634 \mathrm{ff}$.

- bei intracerebralem Hämatom 263

- bei Lindau-Tumor 270

- bei Lösungsmittelintoxikation $343 \mathrm{f}$.

- bei Lues 240

- bei Morbus Wilson $928 \mathrm{ff}$.

- bei neuromuskulären Erkrankungen $837 \mathrm{ff}$.

- bei SAB $256 \mathrm{ff}$.

- bei Syringomyelie 968

- bei Thalamushämatom 267

- bei Thalamusinfarkt 187

- beim toxischen Speiseölsyndrom 350

- bei Tuberkulom 763 f.

- bei Wernicke-Encephalopathie 460

Cornealreflex 535,543

Couplets $159 \mathrm{f}$.

C-reaktives Protein 249,254

Creutzfeldt-Jakob-Erkrankung 247

Cupula 896

$\mathrm{CW}$-Ultraschallgerät 55

-, Doppler-Sonografie 68

Cyanocobolamin 452

Cyclosporin A 570,574

Cytosin-Arabinosid 492,500 ff., 764 ff.

2, 4-D 367

Dapotum $297 \mathrm{ff}$.

DDT 367

Debrisoquin 433

Dehnungsrezeptoren 208

Delir 297,442 f.

Deltaaminolävulinsäure 397

Demenz 92,186 ff.,330,730,917, 931,935

- bei Lösungsmittelintoxikation 330

-, thalamische $186 \mathrm{ff}$.

dentato-oliväres System

Depot-Neuroleptika 530

Depression 917

Depressivitätsskala 513 ff.

Dermographismus 197

Dexamethason 964
191 
Dextran $301 \mathrm{ff}$.

DFP 319

Diabetes mellitus $153,155,208$, $247,276,571,845,901 \mathrm{ff}$.

-, juveniler 571

Dialkylaryltriazene 372

Diazepam 926

Diazoxid $38 \mathrm{f}$.

Dichlorbenzol 366

digitale subtraktionsangiographie (DSA) $133 \mathrm{ff} .197$

Digitalis $10 \mathrm{f}, 22,32 \mathrm{f},, 386$, 595

Dihydralazin $38 \mathrm{f}$.

Dilatation

- der A. subclavia 311

- $\operatorname{der}$ A. vertebralis 311

Dimaval $403 \mathrm{ff}$.

Dinitrobenzol 366

Dinitrophenol 366

Diofan 417

Dioxin $319 \mathrm{f}$.

Diphenhydraminvergiftung $525 \mathrm{ff}$.

Diphenylhydantoin $480 \mathrm{f}$.

disseminierte Neuropathie 364

dissoziierter Hirntod 590,603

Distraneurin $297 \mathrm{ff}$.

Disulfiram 854

DMPS $403 \mathrm{ff}$.

DMPT 375

DNA-Alkylierung $373 \mathrm{f}$.

DNA-Modifikation 373

Dobutamin $647 \mathrm{ff}$.

Dokumentation (Hirntod) $543 \mathrm{ff} .$, $577 \mathrm{ff}$.

Dopamin $595,647,913$

- Rezeptoren 949 ff.

Dopaminergica 911

Doppelbilder $236,247,489,678 \mathrm{f}$.

Doppelmarkertechnik $703 \mathrm{ff}$.

Dopplerfrequenz-Spektren 54,66

-, gepulste 54

Dopplersonographie $54 \mathrm{ff},, 66$, $70,74 \mathrm{ff} ., 79 \mathrm{ff} ., 83 \mathrm{f} ., 92 \mathrm{f} .$, $155,253,275,287,294,304,311$,

$587,603 \mathrm{ff} ., 876,914,956,1005$

-, bidirektionale 197

- beim Hirntod 603 ff.

-, transkranielle $60 \mathrm{ff} ., 96$, $603 \mathrm{ff}$.

D-Penicillamin $361,397,928$

Drogenabhängigkeit $12,420,467 \mathrm{ff}$.

- Endokarditis 12

-, Hirnatrophie $467 \mathrm{ff}$.

$\mathrm{d}_{2}$-Test $475 \mathrm{f} ., 513 \mathrm{ff}$.

Ductus arteriosus apertus $44 \mathrm{f}$.

Duplex-Scan $66 \mathrm{ff}, 79$

Durchblutungsstörung, zerebrale $49,70,79,102,274$

Durchgangssyndrom $292,294 \mathrm{f}$.

Dysarthrie $247,295,392$ f.,587,
729,941

Dysphagie $424 \mathrm{ff}$.

Dyssynergia cerebellaris myoclonica 658

Dystonie 917

Dystrophia myotonica $833 \mathrm{ff}$.

Echoencephalographie beim Hirntod 538

Echokardiographie $3,6,9,16,21$, $29,46,49,51,64,105 \mathrm{ff} ., 111 \mathrm{f}$., $155,197,250,252,276$

-, eindimensionale 49,51 f.,111

-, Kardiomyopathie 21

-, transösophageale $105 \mathrm{f}$.

-, zweidimensionale $102 \mathrm{ff}$.

ECIC-Bypass 64,260

EDTA 260

EEG $180 \mathrm{ff} ., 196 \mathrm{f} ., 290,537 \mathrm{f.,}$ $539 \mathrm{ff} ., 544,556,673,750,754$, $881 \mathrm{ff.}, 886 \mathrm{ff} ., 891,925,932$, $956,995,1005$

- bei Frühgeborenen 556

- beim Hirnstammtod 539 ff.

- beim Hirntod 537 f.,578 ff., $587 \mathrm{ff} ., 625$

-, Nullinien-EEG 544

- bei Pickwick-Syndrom 1008

- bei Schizophrenie 878 ff.

-, Schlaf 197

Ehrmann-Sneddon-Syndrom 252 ff.

Einklemmung 154

- bei Hirnembolie 154

Einschwemmkatheter 204

Einzelfaser-EMG $455 \mathrm{ff}$.

EKG $197,256,276,280,284,297,995$

- bei Friedreich-Ataxie 204 ff.

Elektrolythomoiostase, zerebrale $180 \mathrm{ff}$.

Elektrolytlösung 301

Elektrolytstörungen $408,463 \mathrm{ff}$.

Elektronys tagmographie 287,956

Elektrookulographie 270,901

Elektrophorese 237

ELISA $\ddot{9} 36$

Embolie, s. Hirnembolie, retinale Embolie, Lungenembolie usw.

Embolierezidiv 10,153 ff.

- nach Hirnembolie 154

- bei Vorhofflimmern 10

Emetin $516 \mathrm{ff}$.

EMG $361 \mathrm{ff},, 389 \mathrm{ff},, 399 \mathrm{ff} .$, $446 \mathrm{f} ., 455 \mathrm{ff},, 471 \mathrm{ff}, 0509 \mathrm{ff}$, $547,597,660,663,733,852,869$, $897 \mathrm{ff}$.

Encephalomyelitis disseminata 215

Encephalopathie

-, hypertensive $33 \mathrm{f}$.

-, oaraneoplastische 941

Endangiitis obliterans 254 
enddiastolisches Volumen $27 \mathrm{f}$.

Endokardfibrose 21

Endokarditis 5,12 ff. 51,152

- Anfälle 14

- Embolien 15,50,152

-, infektiöse $13 \mathrm{f}$.

-, Komplikationen 15

-, Mortalität 13

-, mykotisches Aneurysma 15 f.

-, Paresen 14

-, rheumatische

- simplex 250

- Therapie 16

- , Todesursachen 13

Endokarditis eosinophilica Löffler 21

Endokarditis lenta 828

Endoxan 942

ENG $361 \mathrm{ff} ., 399 \mathrm{ff}, 446 \mathrm{ff} .$, $455 \mathrm{ff}, 471 \mathrm{ff} ., 513 \mathrm{ff}$.

Enhancement 232

Enolase $147 \mathrm{ff}$.

Enterokokken 18

Entspannungstherapie 871

Entzugsdelir, alkoholisches $297 \mathrm{ff}, 442 \mathrm{ff}$.

Enzephalitis 13,247

Enzephalopathie $317,330,340 \mathrm{ff}$.

-, Berufskrankheit 330

- Blei $317 \mathrm{f}$.

-, intrathekale Therapie 505 ff.

-, Lösungsmittel $340 \mathrm{ff}$.

Enzymblockade 261

Enzym-Immunoassay $1017 \mathrm{ff}$.

Enzyminduktion durch Antiepileptika 490

EPH-Gestose 931

Epidural-Sonden $139 \mathrm{ff}$.

Epilepsie 14,46,153 f.,196,443, 449,471 ff., 769,933

-, Alkoholismus 443,449

-, Antiepileptikanebenwirkungen $471 \mathrm{ff}$.

- bei der Hirnembolie 154

- des Temporallappens 1014

Erblindung 295

Erbrechen 305

Erethismus 318,328

Ergometrie 72,215

Ergotamin $959 \mathrm{ff}$.

Erythema migrans 744,747

Erythromycin 6

Erythrozytenaggregation $301 \mathrm{f}$.

Ethik 565 ff.

Etomidate $181 \mathrm{f}, 520 \mathrm{ff}, 640 \mathrm{ff}$.

Euthanasie $562 \mathrm{f}$.

- , indirekte 563

Evaluskop 264,267

E-605-Vergiftung 590

Exanthem 489

Exhairese 654 experimentell allergische Enzephalomyelitis (EAE) $806 \mathrm{ff}$.

experimentell allergische Neuritis (EAN) $811 \mathrm{ff}$.

Extrasystolen 158 ff.,197,263

- bei Friedreich-Ataxie 206

-, supraventrikuläre 197

exzentrische Hypertrophie $30 \mathrm{f}$.

Fallot-Tetralogie $44 \mathrm{f}, 169$

Farbsinnstörung $451 \mathrm{ff}$.

faziales EMG 547

Fazialisparese $282,306,658$

Feldmann-Test 725

Fettembolie 608 ff.

Fibrinogenbestimmung 485

Fibrinolysetherapie 63,304 ff.

Fieber 247

Filterbanksystem 55

First Pass Technik $124 \mathrm{f}$

Fisher-Syndrom 281,678 ff.,784

Flow-Mapping 64

Flow-Monitoring, transkranielles 64

Fluoreszenz-Polarisations-Immunoassay $1017 \mathrm{ff}$.

Fluphenazin-Dihydrochlorid $297 \mathrm{ff}$.

Flußdiagramm 61

Flüssigstickstoff 244

Flußprofil, helikales

Fog-Skala 738

Fogging-Effekt 193,637

Folsäuremangel 452,862 ff.

forcierte Diurese 384

Fourier-Transformation $54 \mathrm{f}$., 66,881

foveale Reizung 216

Fragmentation 155

Frequenzabfall 280,283

Frequenzanalyse $61,290,292$

Frequenzbanden 55

Frequenzschwankungen 55

Friedreich-Ataxie 22,204 ff.

- und kardiale symptome $204 \mathrm{ff}$.

- und Lungenfunktion $204 \mathrm{ff}$.

Frühsommermeningoenzephalitis $744 \mathrm{ff}$.

FTA-ABS-Test 239

Funktionsstoffwechsel 176

Furosemid 384,601

GABA $946 \mathrm{f}$.

Galactocerebrosid $811 \mathrm{ff}$.

Gamma-Hydroxybuttersäure $640 \mathrm{ff}$.

Gamma-Kamera 103,124,129,204, 621

Ganglion Gasseri 654,669

Ganglion geniculi $658 \mathrm{ff}$.

Ganglion stellatum 258

Ganzkörperplethysmografie 204 
gastrointestinale symptome

380 ff., $389 \mathrm{ff}, 412 \mathrm{f},, 418$, 424 ff.

Gaumensegelmyoklonus $190 \mathrm{ff}$. Gefäßgeschwulst 270 ff.

Gefäßwandveränderungen

-, arteriosklerotische 51

-, Nekrose 248

Gentamycin 507

Geschmacksstörung 237

Geschwindigkeitshistogramm 66

Gesichtsfelddefekt $451 \mathrm{ff}$.

Gewebszerfallmarker $147 \mathrm{ff}$.

genetische Determination 438

Gerinnungsfaktoren $485 \mathrm{f}$.

Gerinnungsparameter 485

Gewichtsverlust $235,350 \mathrm{ff}$.

Glasgow Coma scale $173 \mathrm{ff}, 557$, 594

Glasgow outcome Scale 148, $173 \mathrm{ff}, 557$

- für Kinder 557

Glaube $566 \mathrm{f}$.

Gliom $963 \mathrm{ff.,976} \mathrm{ff}$.

Glukose-Effekt 430

Glutamat 946

Glycerin $140 \mathrm{f} ., 669,991$

Glykogenanhäufung, intraaxonale 332

Glykogenose $857 \mathrm{ff}$.

Glykogenspeicherung $868 \mathrm{f}$.

Gold 361

$195 \mathrm{~m}$ Gold $124 \mathrm{ff}$.

Granulome, perivaskuläre 248

Guillain-Barré-Syndrom 23,678

HAES $301 \mathrm{ff}$.

Hagen-Pouiseulle-Gesetz 67

Hallervorden-Spatz-Krankheit 931

Halluzination 247,851

Halluzinogene 469

Halogenkohlenwasserstoff $325 \mathrm{f}$.

Haloperidol 926

Haltereflex 480

Hämangioblastom, s. Lindau-Tumor

Hamartom 1014

Hämatokrit $44 \mathrm{f}, 301$

-, kritischer wert 44

Hämatom

- , intramurales 84

-, spontanes intracerebrales 263 ff.

-, Volumen 264

Hämodialyse $385,513 \mathrm{ff}$.

Hämodilution $301 \mathrm{ff}$.

Hämofiltration $513 \mathrm{ff}$.

Hämoperfusion 385,527

Hämorrhagie, sekundäre 232

hämorrhagische Diathese 264

hämorrhagischer Infarkt 155
Hämostase 254

Harnstoff $263 \mathrm{f}$.

Hatchinski Ischämie Skala 115 f. Hautkolorit 395

Hautoberflächenlipide 905 ff.

HAWIE 724

HbS-Antigen 165

Helfer-T-Lymphocyten $764,804 \mathrm{f}$.

Hemianopsie $153 \mathrm{ff}, 252,964$

- bei Hirnembolie 154

- bei Lues 239

Hemiataxie 295

Hemiblock 274

Hemiparese 252,891,964

-, motorische $144 \mathrm{f}$.

Hemmkörper 485 ff.

Heparinisierung $16,62,280$

Hepatitis $778 \mathrm{ff}$.

Herdenzephalitis 797

Herdsymptome, zerebelläre

271

Herniation 629

Herpes simplex 247

- Enzephalitis $750 \mathrm{ff}$.

Herz-Atem-Stillstand 535

Herzbinnenraums zintigrafie

204

Herzfehler

-, angeborener $44 \mathrm{ff}$.

- rheumatischer 152

Herzfrequenzvariabilität 208 , $594 \mathrm{ff}$.

Herzhypertrophie 257

Herzindex $29 \mathrm{f}$.

Herzinfarkt $152,212,252,254$, $263,274,276,294$

Herzinsuffizienz $16,47,212$

Herzkatheter $27 \mathrm{f}, 46$

Herz-Kreislauf-Versagen 481, $639 \mathrm{ff}$.

Herzminutenvolumen 277

Herzmuskelerkrankung bei Friedreich-Ataxie 206

Herzoperation $72,102,263,294$

Herzrhythmusstörungen $151 \mathrm{ff.,}$ $158 \mathrm{ff}, 206,211 \mathrm{f}, 250,274 \mathrm{ff}$. $280 \mathrm{f} ., 290,294$

Herzschrittmacher, s. Schrittmacher

Herztransplantation $571 \mathrm{f}$.

Herzversagen 263

Herzwandperforation 280

Hexacarbon $331 \mathrm{ff}, 365$

Hexachlorophen 366

2,5-Hexandion $331 \mathrm{ff}, 365$

Hexamethylenamin 496

Highlighting $834 \mathrm{f}$.

Hirnabszeß $14,44 \mathrm{f}$.

Hirnatrophie 330,467 ff.

Hirnbasisarterien 60,64

Hirnbiopsie 750

Hirnblutung $37 \mathrm{f}, 264,556 \mathrm{f}$., 577 
Hirnblutung

- bei Frühgeborenen 556

Hirndruck $139 \mathrm{ff} ., 270,550 \mathrm{f.,}$ 980

-, Druckdynamik bei Kindern 557

- und Hirntod 550

-, Messung, epidurale 139 ff. $550 \mathrm{f}$.

-, Steigerung 271

Hirndurchblutung $119 \mathrm{ff} ., 124 \mathrm{ff}$. $277,293 \mathrm{f.}, 639 \mathrm{ff} ., 986 \mathrm{ff} ., 1003$

-, Gold 124

-, Xenon $119,252,1022 \mathrm{ff}$.

Hirnembolie $9,15,21,44 \mathrm{f} ., 49 \mathrm{ff}$. $64,79,102,108,119 \mathrm{ff} ., 151 \mathrm{ff} .$, $206,243,294,309$

-, Emboliequellen 54,152

- , Häufigkeit 151

-, kardiale 233,249

-, Mikroembolie 250

-, Prognose $154 \mathrm{ff}$.

-, regionale Gehirndurchblutung $119 \mathrm{ff}$.

-, Symptomatik $152 \mathrm{ff}$

- , Todesursache 154

Hirninfarkt $70,76,80,83,99$, $105 \mathrm{f.,1} 10 \mathrm{f.,119} \mathrm{ff.,139} \mathrm{ff.}$ $142 \mathrm{ff.,151} \mathrm{ff.,163} \mathrm{ff.,173} \mathrm{ff.}$,

$177 \mathrm{ff} ., 190 \mathrm{f} ., 221,228 \mathrm{ff.}$,

$232 \mathrm{ff} ., 252,254,259,263,274$, $294,301,306,995$

-, Begleiterkrankung 212

-, Brücke 247,294

-, embolischer $5,15,119 \mathrm{f} .$, $151 \mathrm{ff}$.

-, Frühdiagnostik $147 \mathrm{ff}$.

-, Größenmessung 142,148

-, Hirndruck $139 \mathrm{ff}$.

-, Hirnstamm 190 ff.

-, Immunhistologie $243 \mathrm{ff}$.

-, ipsilateraler $76 \mathrm{ff}$.

-, juveniler $163 \mathrm{ff}$.

-, Kleinhirn $190 \mathrm{ff} ., 247,294$

-, lakunärer $142 \mathrm{ff}$.

-, Liquorenzyme $147 \mathrm{ff}$.

- und Migräne 113

-, Mittelhirn 294

-, oberflächlicher $142 \mathrm{ff}$.

-, Prognose $173 \mathrm{ff}$.

-, Schwangerschaft 164

- bei vertebro-basilären verschlüssen $177 \mathrm{ff}$.

-, zentraler $142 \mathrm{ff}$.

Hirnnekrose 259

Hirnödem $139 \mathrm{ff}, 640 \mathrm{ff}, 853$, 991

-, Hirninfarkt $139 \mathrm{ff}$.

hirnprotektive Maßnahmen 147, $639 \mathrm{ff}$.

Hirn-Sequenz-Szintigraphie 197

Hirnstamm $88 \mathrm{ff}, 177 \mathrm{ff}, 190 \mathrm{f} .$,
$304,458 \mathrm{ff} ., 634 \mathrm{ff} ., 992$

-, Blutung $458 \mathrm{ff}$.

-, Insult 305

-, Ischämie 309

-, Hypodensität $634 \mathrm{ff}$.

-, Läsion 277

Hirnstammfunktion $537 \mathrm{ff}$., $582 \mathrm{ff} ., 647$

Hirnstammootentiale 480

Hirnstammtod 539 ff.,556,587 ff.

Hirnszintigrafie $232,649,763$

Hirntod $535 \mathrm{ff} ., 543 \mathrm{ff}, 554 \mathrm{ff} .$, $559 \mathrm{ff} ., 565 \mathrm{ff} ., 577 \mathrm{ff} ., 582 \mathrm{ff} .$, $621 \mathrm{ff}$.

-, Dokumentation $577 \mathrm{ff}$.

-, ethische Aspekte 656 ff.

-, juristische Aspekte $559 \mathrm{ff}$.

- bei Kindern $554 \mathrm{ff}$.

-, Symptome $582 \mathrm{ff}$.

Hirntumor 240

HIS-Bündel-Elektrographie 10

Histokompatibilität 570

HLA-Antigene $711 \mathrm{ff}$.

Hochdruckflüssigkeitschromatografie $695 \mathrm{f}$.

Hochdruckherz $26 \mathrm{ff}, 32 \mathrm{f}$.

-, Stadien 32

-, Therapie 32

Hochdruckkrisen $33 \mathrm{ff}$.

hormonelle Faktoren 84

Horner-Syndrom $236,268,913$

Hörnerv $88 \mathrm{f}$.

Hornhauttransplantation 573

Hörschwelle 88

-, Verschlechterung 481

H1-Rezeptorenblocker 525 ff.

Hüllkurve 55

Hustenreflex 539

HWS-Syndrom 953

Hyalinose 145

Hydrazinderivate 372

Hydrocephalus internus $271,430 \mathrm{f}$. Hydrocephalus occlusivus 47

Hydroxylierungsdefekt $433 \mathrm{ff}$.

Hypakusis 496,658

Hyperextension 84

Hyperhidrosis $197,351 \mathrm{ff}, 365$, $367,413,851$

Hyperkalzämie 320

Hyperkeratosen 403

Hyperlipidämie 153,228 ff.

Hypersensitivitätsangitis 244

Hypersexualität 338,413

Hypersomnie $188 \mathrm{f.,1008 \textrm {ff } .}$

Hyperthermie 215

Hypertonus 5,26 ff.,47,81,142 f. $153 \mathrm{f} ., 197,206,212,228 \mathrm{ff}$. $247,252,256$ ff., 267,276,290, 953

- und intracerebrale Massenblutung 264 
Hyponatriämie 464

Hypothermie $535,543,579$ f.,601, 615

Hypothyreose 914

Hypotonie $212,601,913$

hypoxämische Anfälle $44 \mathrm{ff}$.

Hypoxie 481

Hypoxietoleranz 554

hypoxische Krise 198

Idiosynkrasie 323

IgA-Globulin 244

IgG-Globulin $245,247,349,799 \mathrm{f}$., 820

- bei Lues 240

IgG-Index $785 \mathrm{f}$.

IgM-FTA-ABS-Test 239

IgM-Globulin 245,252

Ileus $380 \mathrm{ff}$.

Immunelektrophorese $254,785 \mathrm{ff}$.

Immunfixation 799 ff.

Immunfluoreszenzmikroskopie $252,772,776$

Immunkomplexpolyneuritis $775 \mathrm{ff}$, 781

Immunneuropathien $788 \mathrm{ff}$.

immunologische Diagnostik 164 , $244,781,784 \mathrm{ff}, 788 \mathrm{ff}$.

- beim Hirninfarkt $243 \mathrm{ff}$.

- bei peripheren Neuropathien 775,784 ff.,788 ff.

Immunperoxidasefärbung 801

Immunsuppressiva $245,502,570 \mathrm{ff}$, 574,852

Immuntherapie 971

Immunvaskulitis $237,243 \mathrm{f},, 249$, $770 \mathrm{ff}$.

Impedanz-Audiometrie

667

Indometacin 260,914

Infarktvolumen 142

Infiltrat 252

Influenza 849

INH $762 \mathrm{ff}$.

Injektion, epidurale 224

Insektizide $319,367,590$

Insomnie 851

Intelligenzminderung bei MS 724

Intensivmedizin $561 \mathrm{ff}, 567 \mathrm{f}$., $590 \mathrm{f} ., 594,598 \mathrm{ff}, 613 \mathrm{ff} .$, $647 \mathrm{f}$.

Interaktion $322 \mathrm{f}$.

Interkostalmuskulatur 206

Interpeak-Latenz $89 \mathrm{f}, 312$, $480 \mathrm{f}$

Intervall, freies $83 \mathrm{f}$.

Intervallhistogramm 897

Intimariß 84

Intimaverdickung 254

Intoxikation $317 \mathrm{ff}, 324 \mathrm{ff}$, $340 \mathrm{ff}, 361$

- Arsen 361,403
-, Blei $317 \mathrm{f} ., 325 \mathrm{f} ., 361 \mathrm{f.,}$ $395 \mathrm{ff}$.

- Dioxin 319

-, Diphenhydramin 525 ff.

- E 605590

-, gewerbliche 317 ff.

-, Insektizide 319,367

-, Kohlenmonoxid 326,364

-, Lithium $407 \mathrm{ff}$.

-, Lösungsmittel 329,340 ff.

- Mangan 320

- Neuroleptika 528 ff.

-, Organophosphate 319

-, Organozinn 336 ff.

-, Phenytoin 471

-, Quecksilber 318,329

-, Schwefelkohlenstoff 364

-, Schwermetalle $324 \mathrm{ff}$.

-, Speiseöl 348 ff.

-, Thallium $324,362 \mathrm{f.,380} \mathrm{ff.,}$ $389 \mathrm{ff.}, 392$.

-, Thymoleptika $525 \mathrm{ff}$.

intrathekale Therapie $500 \mathrm{ff}$. $505 \mathrm{ff}$.

intravenöse Subtraktionsangiographie (ISA) 133

intrazerebraler Druck (ICP) $139 \mathrm{ff}$.

Intrinsic Cardiac Reflexes 596

Intubation 280

Iontophorese $871 \mathrm{ff}$.

Iopamidol $509 \mathrm{ff}$.

irreguläre Hypertrophie $29 \mathrm{ff}$.

Irreversibilität beim Hirntod 544,560

Isaac-Syndrom 854

Ischämie, zerebrale 49,98

isoelektrische Fokussierung $795 \mathrm{ff}$.

Isotopenanreicherung 232

Ixodes ricinus $746,747 \mathrm{ff}$.

Jackson-Anfall 891

Janetta, Operation nach 654

Jervell-Lange-Nielson-Syndrom 10

$123 \mathrm{Jod} 129$

Jodoamphetamin 130

Kalium $263 \mathrm{f}$.

Kalium-Eisen-Hexacyanoferrat $383 \mathrm{f}$.

Kaliumpermanganat $412 \mathrm{ff}$.

Kälteagglutinin 254

Kalzium 295

Kalziumantagonisten 595

Kalzium-Trinatriumpentat 415

Kammerflimmern 276,294 f.

Kanzerogenese $371 \mathrm{ff}$.

Kaposi-Hirnmetastasen 754

Kapselinfarkt 144,148 
Kardioinhibition 198

kardiologische Diagnostik 7,70

- Indikation $6 \mathrm{f}$.

Kardiomegalie 102

Kardiomyopathie $20 \mathrm{ff}, 50,152 \mathrm{f} .$, 868

-, dilative 22

- und Hirnembolie $152 \mathrm{f}$.

-, hypertrophische 21

-, latente 21

Kardioversion 10

Karmansche Wirbelstraßen 63

Karnitinmangelsyndrom 23

Karotisdesobliteration 77

Karotisdruckverfahren 197

Karotisdruckversuch 283,1006

Karotismassage 280

Karotissinus

-, hypersensitiver $9 \mathrm{f}, 274$, $283 \mathrm{ff}$.

-, Massage des 197

Karotissinussyndrom $287 \mathrm{ff}$.

-, hypersensitives 274

Karotissiphon $60,62,76$

- verschluß 306

Karotisstenose $75 \mathrm{ff}, 106$, $129 \mathrm{ff} ., 137,144,167 \mathrm{ff}, 275$

-, supraklinoidale $167 \mathrm{ff}$.

Karotisstumpfdruck 99

Karotistrauma 85,163

Karotisverschluß $129 \mathrm{ff}, 144$

-, bilateraler traumatischer $83 \mathrm{ff}$.

-, embolischer 154

-, SPECT $129 \mathrm{ff}$.

Karotis-Vertebraliserkrankung, kombinierte 76

Karpaltunnelsyndrom 872

Katecholamine 294,299

- , Erhöhung 257

Katheterbiopsie, endomyokardiale 22

Kayser-Fleischer-Ring 928

Kearns-Sayre-Syndrom $22 \mathrm{f}$.

Kernspintomografie, s. NMR

Kichererbsen 320

Kinking 67

Kipptischuntersuchung 197,224

Klappenersatz $16 \mathrm{f}$.

- Endokarditis 17

Klappenfehler $3 \mathrm{ff.,15} \mathrm{ff.,52,}$ 152

Kleinhirnbrückenwinkeltumor 654

Klick, spätsystolischer 107

Klickreiz 88

Klimakterium $228 \mathrm{f}$.

Klippung 16

-, mykotisches Aneurysma 16

Koagulopathie, disseminierte intravasale 250

Kochsalzaufnahme 228 kognitionsabhängige Komponente $92 \mathrm{ff}$.

Kohlenmonoxidvergiftung 326,364

Kojewnikoff-Epilepsie 154

Kollagenase $775 \mathrm{ff}$.

Kollagenose 222

Kollateralversorgung 98

Koma 84,178 f.,613 ff.

Komplikationen

-, kardiale 280

-, respiratorische 280

-, thromboembolische 280

Konakion 487

Kontraktilitätsstörung 52

Kontrastmittel $133 \mathrm{ff}, 232$

Kontrazeptiva 5,164 f.,249

Konzentrationsfähigkeit $474 \mathrm{f}$. $513 \mathrm{ff}$.

konzentrische Hypertrophie $29 \mathrm{ff}$.

Kopfnystagmus $895 \mathrm{ff}$.

Kopfschmerzen $14,34,39,47$,

$153 \mathrm{ff} ., 749,891,980$

- bei Arteriitis temporalis $235 \mathrm{ff}$

-, Diagnostik $952 \mathrm{ff}$.

-, Endokarditis 14

- bei granulomatöser Angiitis 247

- bei Hirnembolie $153 \mathrm{f}$.

-, Hypertonus 34

- bei Lindau-Tumor 272

- bei Lues 240

-, postpunktionelle $224 \mathrm{f}$.

Körnerzelldegeneration 493

Koronarangiografie $70,72,253$

koronare Herzkrankheit $27 \mathrm{ff}$., $70 \mathrm{f} ., 212,274,284$

Koronarfaktor $26 \mathrm{ff}$.

Korpuskelgeschwindigkeit

Korrelationsanalyse 485

kortikales Potential 620

kortiko-nigrale Projektion $946 \mathrm{ff}$.

Kortison $237,247,252,254,502$, $570,574,684,719$ ff.,849

Kortisol 914

Kostennutzenanalyse 52

Krampfanfall 248,283

-, generalisierter 192

Kreatinin $263 \mathrm{f}$.

Kreatinkinase-Isoenzym BB (CKBB) $147 \mathrm{ff}$.

Kreislaufhomöostase, perioperative 98

Kreislaufregulationsstörung $225 \mathrm{f}$.

Kryoglobuline 165,254

Kussmaul'sche Atmung 46

Labyrinthausfall 252

Lagerung 140 
Lagerung

- bei Hirndruck 140

Lambert-Eaton-Syndrom 941

Langzeitantikoagulation 102

Langzeit-EKG $9 \mathrm{f} ., 155,158 \mathrm{ff}$. 277

Lathyrismus 320

Lavage, enterale $385 \mathrm{f}$.

L-Dopa 415,911

Leberenzyme 254,442

Lebertransplantation $571 \mathrm{ff}$.

Leigh-Syndrom 23

Leitungsblock 816

Lemniscus lateralis $88 \mathrm{f}$.

Lentasepsis 45

Leukotrien 824

Levodopa

- und Herzrhythmusstörung 11

- und kardiovaskuläre Erkrankungen $211 \mathrm{ff}$.

Lhermitte 267

limbisches System $337 \mathrm{ff}$.

Lindau-Tumor $270 \mathrm{ff}$.

Linksherzversagen 276

Lipid-hydroperoxide 261

Lipidose 437

Liquor $14 \mathrm{f}, 147 \mathrm{ff}, 180 \mathrm{ff} .$, $252,259,658,679,729,737,747$, $749,751,754,785$ ff., 795 ff., $803 \mathrm{ff}, 846,909,932,935 \mathrm{ff}$. 980

-, Azidose 261

-, Diagnostik bei Hirninfarkt $180 \mathrm{ff}$.

-, Druckschwankungen 224

-, Endokarditis $14 \mathrm{f}$.

-, Enzyme $147 \mathrm{ff}$.

-, Gesamteiweiß 232,247

- bei granulomatöser Angiitis 247

-, isoelektrische Fokussierung $795 \mathrm{ff}$.

- bei Lues 240

- bei Migräne 956 ff.

- bei peripheren Neuropathien $785 \mathrm{ff}$

-, Pleozytose $956 \mathrm{ff}$.

-, Rasterelektronenmikroskopie 792 ff.

-, Unterdruck 224

-, zelluläre Differenzierungsantigene $803 \mathrm{ff}$.

-, Zirkulationsstörung 259

Liquorenzyme $147 \mathrm{ff.,180} \mathrm{f.}$

Liquorzytologie $769,792 \mathrm{ff}$, $803 \mathrm{ff}$.

Lithium 11,407 ff.

- und Herz 11

-, Intoxikation $407 \mathrm{ff}$.

Livedo racemosa $249 \mathrm{ff}, 252 \mathrm{ff}$.

Locked-in Syndrom $88 \mathrm{ff}$.
Long loop-Reflex bei MS $733 \mathrm{ff}$.

Lösungsmittel $329 \mathrm{f} ., 340 \mathrm{ff}$.

Love-Kanalaffäre 320

Lues $239 \mathrm{ff} ., 252$

-, cerebri 239

- , connata 239

-, Meningoradikulitis 784

-, Quecksilberschmierkur 318

-, vaskuläre 239 ff.

Lumbalpunktion $17,243,256$

-, Endokarditis 17

- und postpunktionelle Beschwerden $224 \mathrm{ff}$.

Lumboischialgie 845,871

lumbosakrale Plexusneuropathie $845 \mathrm{ff}$.

Lungentransplantation $572 \mathrm{f}$.

Lupus erythematodes $163 \mathrm{ff}$., 243,489

Lyme disease $744,747,749$

Lymphadenopathie-Syndrom

753

Lymphom $804 \mathrm{f}$.

Lymphozyten-Klone $973 \mathrm{f}$.

Lyse, spontane 103,155

Makroangiopathie, okkludierende 60

Makrozirkulation 301

maligne Hyperthermie 23

Manganvergiftung $320,325,412 \mathrm{ff}$.

Mannit $140 \mathrm{f} ., 384$

Marcumarisierung $51,62 \mathrm{f}$.

Masseterreflex $608 \mathrm{ff}$.

Masse-Volumen-Relation $26 f$.

Mastdarmstörung 222

MBK $331 \mathrm{ff} .365$

McArdle-Syndrom $857 \mathrm{ff}$.

Mediaastverschlu $\beta \quad 155$

Mediahypertrophie $35 \mathrm{f}$.

Medianekrose, idiopathische 169

Medianusneuropathie 366

Medianus-SEP $828 \mathrm{ff}$.

Medianusstimulation $98 \mathrm{f}$.

Mediateilinfarkt $154 \mathrm{ff}$.

Mediaverschluß $155 \mathrm{f}$.

Medulloblastom 976

Mees'sche Querstreifen 361,363 , 403

Megadolichobasilaris 61

Mehrorganentnahme $572 \mathrm{f}, 645 \mathrm{ff}$.

Meige-Syndrom $662 \mathrm{ff}$.

MEK 365

Melkersson-Rosenthal-Syndrom 244

Melphalan 496

Meningitis $13,505 \mathrm{f}, 1773,800$

Meningoenzephalitis, lymphozytäre 737

Meningomyeloradikulitis $747 \mathrm{ff}$.

Meningopolyneuritis 747 
Merettichperoxidase 232

Messkopf 66

Mestinon 683,695

Metastase $977 \mathrm{f}$.

Metenkephalin 909

Methotrexat $492,506,806 \mathrm{ff}$.

Methylbromid 365

Midodrin 916

Migraine accompagnée $61,110 \mathrm{f}$.

Migraine basilaire 61

Migräne $61,110 \mathrm{ff},, 770,871,953$, $956 \mathrm{ff} .959 \mathrm{ff}$.

- und Mitralklappenprolaps $110 \mathrm{ff.}$

- bei progressiver Sklerose 770

Mikroangiopathie, zerebrale $60 \mathrm{f}$. 312

Mikrozirkulation 301

Milzembolie 18

Minamata-Krankheit 318

Minderperfusion

-, cerebrale 96

-, retinale 215

Mipafox 319

Mitralannulus-Verkalkung $6,49 \mathrm{ff}$.

Mitralinsuffizienz $49 \mathrm{ff}$.

Mitralklappe $12 \mathrm{f}$.

- vitium 102

Mitralklappenprolaps $3 \mathrm{ff}$, $49 \mathrm{ff.,105}$ ff.,152,249 f.

- , Hirnembolie 152

-, Inzidenz 108,110

- und Migräne $110 \mathrm{f}$.

mitralkonfiguriertes Herz 50

Mitralsegel 250

Mitralstenose $49 \mathrm{ff}, 250$

Mittelhirn 267

Moja-Moja 243

Monitoring, intraoperatives $98 \mathrm{ff}$.

Monoparese 153

Morbus Alzheimer 92,935

Morbus Behcet 244

Morbus Fabry $22 \mathrm{f}$.

Morbus Fahr 212

Morbus Huntington $917 \mathrm{ff}$.

Morbus Parkinson $909 \mathrm{ff}$.

Morbus Wilson $928 \mathrm{ff} ., 935$

Mortalität

- Endokarditis 13

- Hirnembolie 154

Morvan-Syndrom $851 \mathrm{ff}$.

Multifactorial Index of cardiac risk 263

Multiinfarkt

-, Demenz 96

-, Syndrom 249

Multiple Sklerose $654,715 \mathrm{ff}$.

$719,724 \mathrm{ff} ., 728 \mathrm{ff} ., 733 \mathrm{ff}$.

$737 \mathrm{ff} ., 740 \mathrm{ff} ., 795 \mathrm{f.}, 800$

935,1013

Multiplet-Entladung 852
Musical Murmurs 63

Muskelaktionspotential 815

Muskelatrophie

-, Kugelberg-Welander 839

- , X-chromosomale 828

Muskelbiopsie $249,833,869$

Muskeldystrophie 22

Muskelphosphorylase $857 \mathrm{ff}$.

Muskelpolster 84

M. tensor tymoani

Mutismus 192,268

Myambutol $762 \mathrm{ff}$.

Myasthenia gravis $683 \mathrm{ff}$, $687 \mathrm{ff} .691 \mathrm{ff}, 695,699 \mathrm{ff}$.

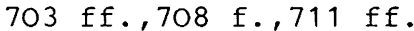

myelin-assoziiertes Glykoprotein $788 \mathrm{ff}$.

Myelographie 225,509 ff.

Myelomalazie $221 \mathrm{ff}$.

Myeloneuropathie 367

mykotisches Aneurysma $13 \mathrm{ff}$.

Myokarddegeneration 204,571

Myokardfaktor $26 \mathrm{ff}$.

Myokardinfarkt $70,102,152$

Myokarditis 152

Myoklonus $190 \mathrm{ff}, 247,295,520$, 583

Myokymie 851

Myopathie $10 \mathrm{f.,22} \mathrm{f.,516} \mathrm{ff}$, 941

-, Herzbeteiligung $10 \mathrm{f}$.

-, toxische 516 ff.

Myopie 281

Myositis fibrosa 22

Nachblutungsrisiko $256 \mathrm{ff}$.

Nackenabdominalreflex $582 \mathrm{f}$.

Nadelelektroden 598

Naevus flammeus 192

$\mathrm{Na}-\gamma-\mathrm{OH}$-butyrat 181

Naloxon 910

Natriumjodidlösung 363

Natrium-Nitroprussid 986

Natriumzitrat 397

Nebenwirkungen

-, Antiepileptika 480

-, Zytostatika $500 \mathrm{ff}$.

Nemaline Myopathie 23

Nembutal-Narkose $480 \mathrm{f}$.

Nervenaktionspotentiale $828 \mathrm{f}$.

Nervenleitgeschwindigkeit 815 ff. $831,847,852,862,868$, $901 \mathrm{ff}$.

Nervensystem

-, parasympathisches 281

-, sympathisches 281

-, vegetatives 208

Nervus ischiadicus 864

Nervus oculomotorius 901

Nervus opticus

-. Atrophie 295 
Nervus opticus

- , Leitungsverzögerung $215 \mathrm{ff.}$ Nervus peronaeus 295,846 f.

Nervus radialis 295

Nervus suralis 822

Nervus tibialis 815

Nervus ulnaris 295

Netzhautablösung 281

Neugeborenenmortalität $554 \mathrm{f}$. neuralgische Schulteramyotrophie 846

Neuritis, chronisch rezidivierende 815 ff.

-, elektrophysiologische Untersuchungen $815 \mathrm{ff}$.

neuro-axonale Dystrophie $931 \mathrm{ff}$. Neuroleptika 297,528 ff.

Neurolues 239

Neuromyopathiesyndrom 349 f., 437

Neuromyotonie $851 \mathrm{ff}$.

neuronale Dysfunktion $180 \mathrm{ff}$.

Neuro-onkologie $371 \mathrm{ff}$.

Neuropathie

-, autonome $208 \mathrm{ff.,360} \mathrm{ff.,}$ 505

- , bulbospinale hereditäre motorische 828 ff.

- , ischämische 295

Neuropeptide $824,909,935$

Neurotransmitter $662,824,843$, 909,935

-, Stoffwechsel 866

$\mathrm{n}$-Hexan $325,331 \mathrm{ff}, 365$

nicht demyelinisierende Erkrankungen 215 ff.

Nicht-Target-Reiz 93

NICOLET CA 100098

Nierenschaden, toxischer 14

Nierentransplantation $570 \mathrm{ff}$., $645 \mathrm{ff}$.

Nifedipine $38 \mathrm{ff}$.

Nitroglycerin $38 \mathrm{f}$.

Nitrosamide $371 \mathrm{f}$.

NMPTP $420 \mathrm{ff}$.

NMR $673,679 \mathrm{f},, 729,750,928 \mathrm{ff.}$, $1012 \mathrm{ff}$.

Non-Uhthoff $216 \mathrm{ff}$.

Noradrenalin $277,647 \mathrm{ff}, 914$

- Spiegel 277

no-reflow-Phänomen 640

Nor-Epinephrin 913

Normtherapie 592

Nortryptilin 433

Notstand $563 \mathrm{f}$.

Nucleolysin 775 ff.

Nucleus cochlearis $88 \mathrm{f}$.

Nucleus olivaris superior $88 \mathrm{f}$. Nucleus pulposus prolaps $775 \mathrm{f}$. Nulldurchgangszähler 54

Nullinien-EEG $536,577,591$,
$603 \mathrm{f} ., 627$

Nystagmus $271,292,480,489,678$, $920,932,941$

O6-Alkylguanin 376

Oberflächenelektrode

88

Ocular-Flutter 758

oddbal1-Paradigma 93

Ohnmacht 196 ff.

$1 / 3$ Oktave-Spektrumanalyse 55

okulocephaler Reflex 535 f., 543

okulovestibulärer Reflex 537

Olbert-Ballon-Katheter 309

Oligodendrogliom 963,976

oligoklonale Bande 659,751, 784 ff.

- bei Lues 240

- bei peripheren Neuropathien $785 \mathrm{f}$.

Olivenhypertrophie 191

Operation $16 \mathrm{f}, 45 \mathrm{f}$.

- Abszeß 16

-, angeborene Angiokardiopathie 45

- Indikation 72

-, mykotisches Aneurysma 72

Ophthalmodynamographie 280

Ophthalmoplegie $671 \mathrm{ff}, 678$

Opioide $469,909 \mathrm{ff}$.

Opsoklonus 758

Optikusatrophie $235,247,393$, 418

-, toxische 393,418

Organentnahme 645 ff.

Organkonservierung 563,645 ff.

Organophosphate 319,367

Organspende $624 \mathrm{f} ., 645 \mathrm{ff}$.

orofaziale Dystonie 662

oropharyngealer Reflex 537,543

Orthostase 212

- Manöver 280

Ösophagusfunktionsstörung 424 ff.

Ösophagusmanometrie $424 \mathrm{ff}$.

oxcárbazepin 886

Panarteriitis nodosa 223,243 , 249

Pancuroniumbromid 297

Pankreastransplantation $572 \mathrm{f}$. Pankreatitis 295

Papillenödem bei Leus 240

Papilitis 239

PAP-Methode 822

paradoxe Embolie $152 \mathrm{ff}$.

Parkinson-Syndrom $211 \mathrm{ff}, 292$,

$417 \mathrm{ff}, 420 \mathrm{f},, 528 \mathrm{ff},, 897$,

$905 \mathrm{ff}, 935,947$

- und kardiovaskuläre Erkrankungen $211 \mathrm{ff}$.

- und Shy-Drager 913 
Parkinson-Syndrom

- unter Neuroleptika 528 ff.

Pathfinder-II-System 204

Patiententestament 562,573

Peak 88 ff.

-, Latenz 93

pektanginöse Beschwerden 70

Penicillin 748

-, Nebenwirkungen 240

-, Prophylaxe $5 \mathrm{f}$.

-, Therapie bei Lues 240

Pentachlorphenol 366

Perfusionsdruck, zerebraler (CPP) $139 \mathrm{ff}$.

Perfusionsszintigrafie $621 \mathrm{ff}$.

Perhexilin $433 \mathrm{ff}$.

periphere arterielle verschlußkrankheit AVK $70 \mathrm{f}$.

Periphlebitis 247

Perkussionsmyotonie 869

Peroxidase-Antiperoxidase-Methode $976 \mathrm{f}$.

Perphenazin 926

Persönlichkeitsschutz 564

Petechien $14 \mathrm{f}$.

Phäochromozytom 33

Phenacetin 433

Phenformin 433

Phenole 320

Phenothiazine und Herz 11

Phentolamin 39

Phenytoin $433,471 \mathrm{ff}, 489 \mathrm{ff}$, $526,851,925,1017$

Physostigmintherapie 525

Pinozytoserate 234

Pilze 18

Pixel $135 \mathrm{f}$.

Plantarflexion 574,583

Plaques $67,72,312$

-, arteriosklerotische 54,79 ff., 84

-, atheromatöser 295

Plasmapherese 281

P 300-Latenz 92

Plättchenaggregationstest $111 \mathrm{f}$.

Plättchenfaktor $4105 \mathrm{ff}, 165$, 254

Plättchenmarkierung 103

Plättchenszintigrafie 64

-, 111 In $102 \mathrm{ff}$.

Plexus lumbosacralis $845 \mathrm{ff}$.

Pneumothorax 295

Polani-Moynahan-Syndrom 22

Poliomyelitis $837 \mathrm{ff}$.

Polyglobulie $44,270,272$

Polymyalgie $235 \mathrm{f}$.

Polymyositis $22,349 \mathrm{ff}, 516,784$

Polyneuropathie $208 \mathrm{ff}, 326$, $331 \mathrm{ff.}, 360 \mathrm{ff},, 775 \mathrm{ff} ., 845$, 854,942

-, Akrylamid 364

-, Alkohol 442 ff.,455 ff.,459
-, Antiepileptika 471 ff.,489

-, Arsen 361,403 ff.

-, Athylenoxid 364

-, Benzol 366

-, Berufskrankheit $326 \mathrm{f}$.

-, Blei $317 \mathrm{f} ., 327 \mathrm{f}$.

-, CIS-Platin 496

-, diabetische $208 \mathrm{ff}, 901 \mathrm{ff}$.

-, DDT 367

-, entzündliche $785 \mathrm{ff}$.

-, Folsäure 862 ff.

- und Hepatitis $778 \mathrm{ff}$.

-, Hexacarbon $331 \mathrm{ff}$.

-, Immunkomplex-P. $775 \mathrm{ff}$.

-, kausalgische 380

-, Kohlenmonoxid 326,364

-, Kohlenwasserstoff $366 \mathrm{f}$.

-, Mangan 412 ff.

-, Organophosphat $319,327,367$

-, Phenole 366

-, Porphyrie $429 \mathrm{ff}$.

-, Schwefelkohlenstoff 364

-, sensomotorische 209

-, serogenetische $775 \mathrm{ff}$.

-, Speiseölvergiftung $349 \mathrm{ff}$.

-, Thallium $363,380 \mathrm{ff} ., 389 \mathrm{ff}$.

-, Triorthokresylphosphat 366

-, urämische $513 \mathrm{f}$.

-, Vitamin B6 $862 \mathrm{ff}$.

Polyradikulitis $837,839,845$

Polyradikulitis Guillain-Barré $280 \mathrm{ff} ., 768,778 \mathrm{ff}, 784 \mathrm{ff}$.

Polytrauma $297 \mathrm{ff}$.

Polyurie 407

poor metabolizer 433

Porphyrie $429 \mathrm{ff}$.

Porphyrine 397,429 ff.

Positronen-Emissions-Computertomographie $124,127 \mathrm{f} ., 949 \mathrm{ff}$. postpunktionelle Beschwerden $224 \mathrm{ff.}, 502$

Posturografie $941 \mathrm{ff}$.

Potenzstörung 209

PPD 764

Pravidel, s. Bromocriptin

Prednisolon 237

Priapismus 222

primemover 322

Primidon 472 ff.,925,1017

Primitivschablonen 590

Procaine $181 \mathrm{ff}$.

Procarbazin 374,492

procongierter reversibler Insult $106,121,153,169,277$

Prognose der Hirnembolie $154 \mathrm{ff}$. programmierte stimulation 10, 22

progressive Paralyse 239

Promit 301

Propanolol 433,596

Prostacyclin 259

Prostaglandin 824,914 
Proteinanalyse 232

Proteinbiosynthese-Hemmung 517

Protein C 165

Pseudo-Carotis-Thrombose 550

Pseudotabes / Peripherica 368

Pseudotumor cerebri $980 \mathrm{ff}$.

Psoashämatom 845

psychische Symptome 14,21,337, $343 \mathrm{f} ., 392 \mathrm{f}, 418,430,513 \mathrm{ff}$., 525

- Antiepileptika 474 f.

- Dialyse $513 \mathrm{ff}$.

- Endokarditis 14

- latente Kardiomyopathie 21

-, Lösungsmittel-Intoxikation 342 ff.

-, Manganvergiftung 418

-, Organozinn-Intoxikation $337 \mathrm{f}$.

-, Thalliumvergiftung $392 \mathrm{f}$.

Psychophysiologie $995 \mathrm{ff}$.

Psychosyndrom, hirnorganisches $115,252,326,336 \mathrm{ff} ., 344,442 \mathrm{f}$.

-, Alkoholismus 442

-, Lösungsmittel-Intoxikation 342 ff.

-, Organozinn-Intoxikation $336 \mathrm{ff}$.

pulmonalstenose-syndrom $44 \mathrm{f}$. Pulsrepetitionsrate 60

Pupillenreaktion 535 f., 543, $578 \mathrm{f}$.

Purinbasen 376

Purkinje-Zellen 492

Purpura 15

psychopathologische syndrome bei Lindau-Tumor 271

Pyramidenbahnzeichen 247

Pyridostigmin $695 \mathrm{ff}$.

QT

- Intervall 10

- , Verlängerung 258

QT-Syndrom, angeborenes 10

$\mathrm{QT}_{\mathrm{C}}$-Dauer $257 \mathrm{f}$.

Quecksilbervergiftung 318,326 , 329

Querschnittssyndrom $968 \mathrm{ff}$.

Quick-Wert 486

Radioimmunassay 824

Ramsay-Hunt-Syndrom 658 ff.

Rate-Pressure-Product 298

Ratten-Pellagra 864

Rauchen 5,153 f.,451 ff.

Raynaud-Syndrom 770

rCBF 98 ff.

Radicularis-Magna-Syndrom

221

Raynod-Syndrom 212

Real-Time-Bild 67

Reangiographie 106,154
Reanimation $11,120,560$

Rechtfertigungsproblem $563 \mathrm{f.,}$ $565 \mathrm{f}$.

Rechtsfrage $559 \mathrm{ff}$.

Reendothelialisierung 80

Reflex, axialer 57

regionale Gehirndurchblutung $119 \mathrm{ff}$.

Regressionsanalyse, lineare 94

Reiter-Syndrom 244

Rekalzifizierungszeit 485

Rekanalisation 106,155

Rektoskopie 932

REM-Schlaf 888

Renin 914

Residualvolumen 205

retinale Embolie 4

retinale perfusion 219

Retrobulbärneuritis 215,239, $719 \mathrm{ff} ., 729$

Reye-Syndrom 23

Rezidivstenose 312

Rhabdomyolyse 22

rheumatische Herzkrankheit 102 Rhythmusstörungen $9 \mathrm{ff}, 15$, $50 \mathrm{ff.}, 151 \mathrm{ff}$.

RIA 936

Richmond-Schraube 557

Riesenaxonopathie 365

Rifampicin $762 \mathrm{f}$.

Rigor 247,917

riMLF 188

Risikofaktoren $74,81,107 \mathrm{f}$, $143 \mathrm{f} ., 153 \mathrm{f},, 164 \mathrm{f} ., 170$, $178,252,267$

-, kardiovaskuläre 263 ff.

Röntgenbildverstärker 135

Rückenmarksnekrose 337

Ruffles 792

SA-Block 274

Sarkomatose $768 \mathrm{f}$.

Sauerstoffextraktionsrate $127 \mathrm{f}$.

Sauerstoffverbrauch $31 \mathrm{f} ., 127 \mathrm{f}$.

Sauerstofftherapie, hyperbare $168 \mathrm{f}$.

Saure-Maltase-Mangel. $868 \mathrm{ff}$.

Schädel-Hals-Trauma $83 \mathrm{ff}$.

Schädel-Hirn-Trauma 594

Schallauslöschung 67

Schallkopf 81

Schellong-Test 914

Schirmer-Test 658

Schizophrenie $917,947,949$

- und EEG 878 ff.

Schlaf-Apnoe $1008 \mathrm{ff}$

Schlafentzug 196

- und EEG 197

Schlafmuster $886 \mathrm{ff}$.

Schlaganfall, s. Hirninfarkt 
Schleimhautreizung $417 \mathrm{ff}$. Schlucksynkopen $1003 \mathrm{ff}$. Schlundmyoklonien $190 \mathrm{f}$. Schmerz

- bei Arteriitis temp. $235 \mathrm{ff}$.

- der Gelenke 235

- der Glieder 235,247

- beim Kauen 235

Schmerzreiz 543,582 ff.

Schnüffelmißbrauch $331 \mathrm{ff}, 342$

Schock, kardiogener $294 \mathrm{f}$.

Schrankenstörung, s. Blut-LiquorSchranke

Schrittmacher

-, Behandlung 274,527

- Implantation 283,287 ff., 1004

-, Indikation 280

- , Träger $290 \mathrm{ff}$.

Schwangerschaftsabbruch 559

Schwefelkohlenstoff $343 \mathrm{f} ., 364$

Schwefelwasserstoff 363

Schweibtest 914,969

Schwindel 21,34,225,270,272,283, $285,287,305,679,913$

Sedativa 595

Sektorscanner 66

Sepsis $13 \mathrm{f} ., 280,295$

-, Staphylokokken 250

septische Embolie $13 \mathrm{ff}$.

Sermion 216

Serotonin $111,181,911$

Serumkrankheit 849

Serumosmolalität 141

Seveso-Katastrophe 320

Shy-Drager-Syndrom $212,913 \mathrm{ff}$.

Sicca-Syndrom 349

Sick-Sinus-Syndrom 9 f.,274

Signalanalyse 54

Signalfarbtransformation 55

Signalfrequenz 55

Silberfärbung 801

Silylverbindung 809

Single Photon-Emissions-Computertomographie $129 \mathrm{ff}$.

Sinusarrhythmie 276

Sinusknotensyndrom 284

Sinusvenenthrombose $173 \mathrm{ff}$.

-, Prognose $173 \mathrm{ff}$.

Sjögren-Syndrom 244

skapuloperoneales Syndrom 23

Sklerodermie $244,770 \mathrm{ff}$.

Skoliose bei Friedreich-Ataxie 204

Small-Part-Dopplereinschub 66

Snellen-Tafel 216

somatosensorisch evozierte Potentiale $98 \mathrm{ff} ., 399 \mathrm{ff}, 404$, $520 \mathrm{ff},, 592,598 \mathrm{ff} ., 603 \mathrm{ff} .$, $608 \mathrm{ff} ., 617 \mathrm{ff} ., 653 \mathrm{ff}, .679$, $816 \mathrm{ff} ., 868,932,969$
Somatostatin $935 \mathrm{ff}$.

Somatostatin-Analog $886 \mathrm{ff}$.

Somnolenz $153 \mathrm{f} ., 305$

Sopor 154

Sorbit 140

Spannungskopfschmerz

SPECT 129 ff.

Spektrodop 55

Spells 45

spinale Phänomene 579 f.,582 ff.

spinale Schablonen 583

Spinalis-anterior-syndrom $221 \mathrm{ff}$.

Spinalisationszeichen $579,582 \mathrm{ff}$. spinovaskuläre Syndrome $221 \mathrm{ff}$.

Spirochäte 747

Spirometrie 204

Splenektomie $691 \mathrm{ff}$.

sprachstörungen, zerebrale $999 \mathrm{ff}$

Sprachzerfall 247

Standversuch 208

Stapedius-Reflex 667

Staphylococcus aureus 15

Staphylokokken 13 ff.

Status epilepticus 154

Staungspapille $240,270,980$

- bei Lues 240

Steele-Richardson-Erkrankung 212

Stenosegrad 79

Stenosen, asymptomatische $74 \mathrm{ff}$.

Sterbeprozeß 560,567

Sterblichkeitsrate 265

Stichlochdrainage $224 \mathrm{ff}$.

Stimmlippenmyoklonus 190

Strahlen-Detektor-System 621

Streaming 518

Streptococcus viridans 12

Streptokinase 306

Streptokokken $13 \mathrm{ff}$.

Streptomycin 507

Streß 228,257

Striatum, ischämische Läsion 192

Strömungsprofil, laminäres 54 , 58

strukturloses Hirn $628 \mathrm{ff}$.

Strukturstoffwechsel 176

Styrol $327,329 \mathrm{f}$.

Subarachnoidalblutung $14,256 \mathrm{ff}$. 632

-, mykotisches Aneurysma 14

- und Vasospasmus 259 ff.

Subclavian Steal $76 \mathrm{f}$.

Subtraktionsangiografie, digitale 63,83

Such-Like-Syndrom $357 \mathrm{f}$.

Sulcocommissuralis-syndrom 221

supraklinoidale Stenose $167 \mathrm{ff}$.

suxamethoniumchlorid $871 \mathrm{ff}$.

Sympathikomimetika 280 
Sympathikusreizphänomene $380 \mathrm{f}$. Syndrom $x \quad 21$

Synkope $9 \mathrm{ff.,21} \mathrm{f},, 158 \mathrm{ff} .$, $277,283,285,287 \mathrm{ff} ., 1004 \mathrm{f}$.

- bei Herzrhythmusstörungen $9 \mathrm{f} ., 158 \mathrm{ff}$.

- bei Kardiomyopathie $21 \mathrm{f}$.

- , kardiovaskuläre 198

- bei Shy-Drager 913

- vagovasale 196

Syringomyelie

- und NMR 1013

- und spinales Trauma 968 ff.

Tabak-Alkohol-Amblyopie $451 \mathrm{ff}$. Tabes dorsalis 239

Tachykardie, paroxysmale 274

Tachykardie, supraventrikuläre $9,158 \mathrm{f}, 256$

Tachykardie, ventrikuläre $9 \mathrm{f}$. 50,159

Tageszeit $153 \mathrm{f}$.

- Hirnembolie 153

Takayasu-Arteritis

Target 323

Target-Reiz 93

Technetium $124 \mathrm{f}, 129 \mathrm{f} ., 204$, 626

Tensor-Tympani-Reflex 666

Terpentin 340

Tetanus 318

Tetrabenazine 933

Tetrachlorkohlenstoff 365

Tetrazykline 749

thalamische Demenz $671 \mathrm{ff}$.

Thalamus

-, bilateraler 186,192

- Hämatome $267 \mathrm{ff}$.

- Infarkt 186 ff.

- , ischämische Läsionen 192 ff.

Thallium-Szintigraphie 386

Thalliumvergiftung $324,362 \mathrm{f}$. $380 \mathrm{ff}, .383 \mathrm{ff}, 389 \mathrm{ff} ., 392 \mathrm{ff}$.

Thermodilutionsmethode 297

Thermokoagulation 654

Thiopental 184,640

Thrombangitis obliterans 169, $244 \mathrm{f}$.

Thrombembolie $4 \mathrm{f}, 9,9,45 \mathrm{f}$.

Thrombendarteriektomie $84 \mathrm{f}$.

Thromboplastinzeit $485 \mathrm{ff}$.

Thrombose 280

- Basilans- 309

- traumatische 85

-, venöse 221

-, Vertebralis- 309

Thromboxan 259 ff.,824

-, antochthone Entstehung 261

-, Inhibitoren 643

-, Queele 260

-, Synthese 295
Thrombozytenaggregate $110 \mathrm{f}$. $115 \mathrm{ff}$.

Thrombozytenaggregation 301

-, ADP 111

-, induzierte $111 \mathrm{ff}$.

-, Serotonin 111

Thrombozytenaggregationshemmer $5,72,81,108,168$

Thrombozytenanreicherung, pathologische 103

Thrombozytenfunktionsstörung 105 ff., 165

Thrombozytenszintigraphie 52 ff. , 165

Thrombozytenzerfall 260

Thrombusnachweis, intrakardialer $52,102 \mathrm{ff}$.

Thymektomie 691

Thymoleptikavergiftung $525 \mathrm{ff}$.

Thymom $841 \mathrm{ff}$.

Thyreotropin-Releasing-Hormon $841 \mathrm{ff}$.

Tibialis-Anterior-Syndrom 833

Tic doloureux 654

tight junctions 317

Tine-Test 748

Tinnitus $666 \mathrm{ff}$.

T-Lymphozyten 699 ff.,971

Todesursachen

- bei Gefäßpatienten 75

- bei Hirnembolie 154

Todeszeit $559 \mathrm{ff}$.

Token-Test 995

Toluol 327,340

Totalkapazität 206

toxisches Speiseölsyndrom $348 \mathrm{ff}$.

Toxoimunologie $322 \mathrm{f}$.

Toxoplasmose-Enzephalitis $753 \mathrm{ff}$. TPHA

-, Test 239

-, Titer 254

TPI-Test 239

Traceruntersuchung $820 \mathrm{ff}$.

Trachealreflex 543,578

Tracheostomie 1008

Transducer 79

transitorisch ischämische Attacke $5,14,54,70,75,80,83$, $98,106,115$ ff. $130,153 \mathrm{ff} .$, 158 ff., 169 f., 178 ff., 212, $221,249,277,287,304$

- und Herzrhythmusstörung 158 ff.

-, Hirnembolie $153 \mathrm{ff}$.

- und MKP $106 \mathrm{f}$.

- und Plättchenaggregate $115 \mathrm{f}$.

-, supraklinoidale Stenose 169

-, vertebrobasilär $178 \mathrm{ff}$.

Transitzeit $124 \mathrm{f}$.

Transmitter $946 \mathrm{ff}$.

Transplantation $563 \mathrm{f} ., 569$, $570 \mathrm{ff}, 645 \mathrm{ff}$. 
Transposition $44 \mathrm{f}$.

transtemporale Beschallung 60

Transversalsymptomatik bei Lues 239

Transversalsyndrom 221

Traumalokalisation 83

Tremor $247,292,318,407,413,678$, $729,898,913,917$

Tremor merkurialis 318

Treponema pallidum 747

Triaethylzinn (TET) $336 \mathrm{ff}$.

Triamcinolon 505 ff.

-, intrathekale Therapie $505 \mathrm{ff}$.

Triaryl-Phosphate 319

Trichloraethylen $341 \mathrm{f}$.

Triclophenol 320

trigemino-trigeminaler Reflex 669

Trigeminusneuralgie 653 ff.,953

Trikuspidalklappe 12

Trimethaphan 986

Trimethylzinn (TMT) $336 \mathrm{ff}$.

Trinitroglycerol 986

Triorthocresylphosphat 319,367

Tuberkulom 762 ff.

Tuberkulostatika $762 \mathrm{ff}$.

Turbulenz 55,57,67

-, Phänomene $54 \mathrm{f}$.

Tumorblutung 264

Tumorinduktion $371 \mathrm{ff}$.

Tumormarker 254

T-Zell-Linien-Übertragung $806 \mathrm{ff}$.

Übergewicht, s. Adipositas

Úberlebensrate 74

Uhthoff-Phänomen $215 \mathrm{ff}$.

Umweltgift $317 \mathrm{ff.} 360 \mathrm{ff}$.

Ulzeration 80

Urapidil $38 \mathrm{f}$.

Urinstatus 254

Urokinase 306

Vagusreiz 280

Vakzination 849

Valproat $472 \mathrm{ff}, 480 \mathrm{ff},, 1017$

Valsalva

-, Manöver 282

-, Quotient $208 \mathrm{f}$.

-, Reaktion 208

-, Test 208,280

Variationskoeffizient 208

variköse Degenerationen 17

Vasculitis 14,249 ff.,254

-, Endokarditis 14

Vasodepression 198

Vasokonstriktor 259

Vasopressin $980 \mathrm{ff}$.

Vasospasmen 60,259 ff.

VDRL-Test 239

Vena Galeni-Aneurysma $47 \mathrm{f}$.

Vena jugularis 297
Venendruck, zentraler 263

Ventrikel

-, Blutung 267

-, Kontraktilitätsstörung $50 \mathrm{f}$.

- bei SAB 256

Ventrikeldynamik $29 \mathrm{f}$.

Ventrikelstimulation, s. programmierte Stimulation

Ventrikelthrombus 294

Verantwortung $565 \mathrm{f}$.

Verapramil 22

Verbrauchskoagulopathie 295

Verfügungsrecht 569

Verkehrsunfälle 573,577

Verschlußhydrocephalus 270

Vertebralisstenose $144,177 \mathrm{ff}$.

Vertebralisverschluß 144,

$177 \mathrm{ff}$.

vertebrobasiläre Insuffizienz $88,177 \mathrm{ff} ., 277,287,872$

Verwirrtheitszustand 247

verzögerte Neurotoxizität 319

Video-Simultandoppelbild-Aufzeichnung 197

Vierfelder-Tafeln 56

Vigilanztest $513 \mathrm{ff}$.

Vincristin 942

visuell evozierte potentiale $92 \mathrm{ff}, 215,240,311,399 \mathrm{ff}$. $446 \mathrm{f.}, 451 \mathrm{ff} ., 509 \mathrm{ff},, 715$, 719 ff. $, 729,868,932$

Visusminderung 215,452

Vitamin B1 460

Vitamin B6-Mangel $862 \mathrm{ff}$.

Vitamin K 452

Vitaminsubstitution $452 \mathrm{f}$.

Vorderhornerkrankung 828

Vorhofflimmern $9 \mathrm{ff} ., 102 \mathrm{f} .$, $159 \mathrm{ff} ., 274,276,294$

- bei Friedreich-Ataxie 206

-, Hirnembolie 9,50

- und Mitralklappenprolaps 4

Vorhofmyxom 50,163

Vorhofumkehr $46 \mathrm{f}$.

Wachstumshormon 886

Warwick-Schema $672 \mathrm{f}$.

Weber-Syndrom 294

Webster-Scale 905

Wegener'sche Granulomatose 243

Wernicke Enzephalopathie $443 \mathrm{f}$., $458 \mathrm{ff} .463 \mathrm{ff}$.

Windkesselgefäße 230

Xenon $119 \mathrm{ff} ., 125 \mathrm{f},, 626,986$,

$1022 \mathrm{ff}$.

xylol 327,341

Zeckenstich 744,747

Zentralaxterienembolie 216 
zentrale pontine Myelinolyse $463 \mathrm{ff}$.

zentrale Überleitungszeit 98 zerebelläre Degeneration, paraneoplastische $941 \mathrm{ff}$.

zerebelläre Enzephalitis $758 \mathrm{ff}$. zerebelläre Symptome $392 \mathrm{ff}$ zerebelläre Ischämie, s. Durchblutungsstörung, zerebrale zerebrokardiale Dissoziation 596 zerebro-vaskuläre Insuffizienz 115 ff., 212,654 zerebro-vaskulärer InsuLt, s. Hirninfarkt

Zervikalsyndrom 871

Zinnvergiftung $336 \mathrm{ff}$.

Zirkulationsstörung $155 \mathrm{f}$.

$\mathrm{Z}$ isternen 257

Zoster-Meningoradikulitis 784

Zoster oticus 658

Zystadenokarzinom 250

Zytostatika-Toxizität 492 ff. 